MPP-2006-15

\title{
Measuring neutrino masses and dark energy with weak lensing tomography
}

\author{
Steen Hannestad \\ Department of Physics and Astronomy \\ University of Aarhus, DK-8000 Aarhus C, Denmark \\ Huitzu Tu \\ Department of Physics and Astronomy \\ University of Aarhus, DK-8000 Aarhus C, Denmark \\ Yvonne Y. Y. Wong \\ Max-Planck-Institut für Physik (Werner-Heisenberg-Institut) \\ Föhringer Ring 6, D-80805 München, Germany \\ E-mail: sth@phys.au.dk, huitzu@phys.au.dk, ywong@mppmu.mpg.de
}

\begin{abstract}
Surveys of weak gravitational lensing of distant galaxies will be one of the key cosmological probes in the future. We study the ability of such surveys to constrain neutrino masses and the equation of state parameter of the dark energy, focussing on how tomographic information can improve the sensitivity to these parameters. We also provide a detailed discussion of systematic effects pertinent to weak lensing surveys, and the possible degradation of sensitivity to cosmological parameters due to these effects. For future probes such as the Large Synoptic Survey Telescope survey, we find that, when combined with cosmic microwave background data from the Planck satellite, a sensitivity to neutrino masses of $\sigma\left(\sum m_{\nu}\right) \lesssim 0.05 \mathrm{eV}$ can be reached. This results is robust against variations in the running of the scalar spectral index, the time-dependence of dark energy equation of state, and/or the number of relativistic degrees of freedom.
\end{abstract}




\section{Introduction}

With the advent of precision measurements of the cosmic microwave background (CMB), large scale structure (LSS) of galaxies, and distant type Ia supernovae (SNIa), a new paradigm of cosmology has been established. In this new standard model, the geometry is flat so that $\Omega_{\text {total }}=1$, and the total energy density is made up of matter $\left(\Omega_{m} \sim 0.3\right)$ [comprising of baryons $\left(\Omega_{b} \sim 0.05\right)$ and cold dark matter $\left(\Omega_{\mathrm{CDM}} \sim 0.25\right)$ ], and dark energy $\left(\Omega_{\mathrm{de}} \sim 0.7\right.$, with equation of state $\left.w \equiv P / \rho \simeq-1\right)$. With only a few free parameters this model provides an excellent fit to all current observations [1-4]. In turn, this allows for constraints on other, nonstandard cosmological parameters.

Two very interesting possibilities discussed widely in the literature are: (i) a subdominant contribution to the total energy density in the form of neutrino hot dark matter (HDM) and hence limits on the neutrino mass $m_{\nu}$, and (ii) an equation of state for the dark energy component that deviates from $w=-1$ and/or is time-dependent.

In the first case, data from atmospheric and solar neutrino oscillation experiments strongly suggest two important mass differences in the neutrino mass hierarchy, $\Delta m_{\mathrm{atm}}^{2} \simeq\left|\Delta m_{23}^{2}\right| \simeq 2.4 \times 10^{-3} \mathrm{eV}^{2}$ and $\Delta m_{\text {sun }}^{2} \simeq \Delta m_{12}^{2} \simeq 7.9 \times 10^{-5} \mathrm{eV}^{2}$ (e.g., [5]). The simplest interpretation of this data set implies $m_{1} \sim 0, m_{2} \sim \sqrt{\Delta m_{\text {sun }}^{2}}$ and $m_{3} \sim \sqrt{\Delta m_{\mathrm{atm}}^{2}}$, i.e., the so-called normal hierarchy. Alternatively, since the sign of $\Delta m_{23}^{2}$ cannot be fixed by atmospheric neutrino oscillations, an inverted hierarchy with $m_{3} \sim 0, m_{2} \sim \sqrt{\Delta m_{\text {atm }}^{2}}$ and $m_{1} \sim \sqrt{\Delta m_{\text {atm }}^{2}}$ is also allowed. Yet a third possibility is that the three mass eigenstates are nearly degenerate, with $m_{1} \sim m_{2} \sim m_{3} \gg \sqrt{\Delta m_{\text {atm }}^{2}}$. If the last case prevails, then oscillation experiments can offer no useful information on the absolute neutrino mass scale.

Cosmology provides an interesting measure of the neutrino mass through the neutrino's kinematic effects on large scale structure formation (e.g., [6]). Qualitatively, free streaming of a HDM particle causes essentially all of its own perturbations to be erased on scales below its free streaming length $\lambda_{\mathrm{fs}}$. This erasure is simultaneously propagated to other species (eg., CDM) via the metric source term. The net effect on the present day large scale matter power spectrum $P_{m}(k)$ is a suppression of order $\Delta P_{m}(k) / P_{m}(k) \sim-8 \Omega_{\nu} / \Omega_{m}$ (assuming $\Omega_{\nu} \ll \Omega_{m}$, and normalisation at $k \rightarrow 0$ ) at large wavenumbers $k \gg k_{\mathrm{fs}} \sim 2 \pi / \lambda_{\mathrm{fs}}$, where $\Omega_{\nu} h^{2}=\sum m_{\nu} /(92.5 \mathrm{eV})$. Indeed, within the $\Lambda$ CDM framework, LSS observations to date have already been able to constrain the sum of the neutrino mass to $\sum m_{\nu} \lesssim 0.3 \rightarrow 1 \mathrm{eV}(2 \sigma)$ [7-15], depending on the data set used. This limit should be compared with the current laboratory bound from tritium $\beta$ decay experiments, $m_{\nu}<2.2 \mathrm{eV}(2 \sigma)[16,17]$, and the projected sensitivity of the upcoming KATRIN experiment, $\sim 0.2 \mathrm{eV}[18,19]$.

In the second case, the simplest candidate for dark energy is a cosmological constant $\Lambda$, obeying $w=-1$. A minimal extension of this is to assume that the dark energy equation of state is given by $P=w \rho$, where $w$ is constant in time. Using this framework, various cosmological data sets have been analysed in many independent 
studies (e.g., [20-22]). With the most recent data $w$ is constrained to be extremely close to -1 , the value for a pure cosmological constant (e.g., [23]).

However, in many particle physics models of dark energy, the equation of state is expected to depend on the scale factor, and many authors have analysed the data in terms of dark energy models with varying equations of state (e.g., [24-26]). From an observational point of view, the simplest possibility is that $w$ undergoes a transition between two asymptotic values. This is very often also the case in particle physics models of dark energy such as quintessence [27-29]. The same is also true in models where dark energy is a consequence of modified gravity [30,31]. Cosmological data to date show no evidence for time variations in the dark energy equation of state. However, for future high precision observations, it is natural to search for such time dependences. Later on when we analyse the ability of future cosmological probes to constrain neutrino masses and the dark energy equation of state, we will also include this possibility using a simple parameterisation.

Recently, it was shown that presently available data admit an apparent degeneracy between $m_{\nu}$ and $w$ [32]. Specifically, by extending $w$ (taken to be constant in time) into the phantom regime $(w<-1)$, the neutrino mass bound relaxes to $\sum m_{\nu} \leq 1.48 \mathrm{eV}$ from a combined CMB+LSS+SNIa analysis, compared to $\sum m_{\nu} \leq 0.65 \mathrm{eV}$ for the case of a fixed $w=-1$ using the same data set. This $m_{\nu}, w$ degeneracy can be explained as follows. The suppression of power at small scales due to a large $m_{\nu}$ can be accommodated by LSS data if $\Omega_{m}$ is simultaneously increased to "restore" the matter power spectrum to its canonical spectral shape. On the other hand, $w$ and $\Omega_{\mathrm{de}}$ (and hence $\Omega_{m}=1-\Omega_{\mathrm{de}}$ in a flat universe) are degenerate parameters in the Hubble expansion rate at redshifts probed by SNIa, such that an increased $\Omega_{m}$ can be compensated for by a decreased $w$. These two degeneracies then combine to produce an apparent interplay between $m_{\nu}$ and $w$.

It has been shown in [15] that the $m_{\nu}, w$ degeneracy can be effectively broken by adding to the likelihood analysis new data from the Sloan Digital Sky Survey (SDSS) measurement of the baryon acoustic oscillation (BAO) peak [33], bringing the present bound on the sum of neutrino masses to the $0.3 \rightarrow 0.5 \mathrm{eV}$ range. In this work, however, we consider the prospects for breaking this degeneracy with an alternative approach, namely, weak gravitational lensing.

Weak gravitational lensing (or cosmic shear) of distant galaxies by the intervening large scale structure provides an unbiased way to map the matter distribution in the universe (e.g., [34-36]). Perturbations in the density field between the source and the observer induce distortions in the images of source galaxies. By measuring the angular correlation of these distortions, one can in principle reconstruct the underlying three-dimensional matter power spectrum, without making assumptions about the luminous versus dark matter bias (cf. conventional galaxy surveys). Since its first detection in 2000 [37-40], the sensitivity of weak lensing surveys has improved to the stage where they are able to provide constraints on the matter density $\Omega_{m}$ and the fluctuation amplitude $\sigma_{8}$ that are competitive with other cosmological probes (e.g., $[41,42])$. Several larger surveys are now in operation or in planning. These 
include the Canada-France-Hawaii Telescope Legacy Survey (CFHTLS) [43], the Dark Energy Survey (DES) [44], Pan-STARRS [45], and VISTA [46], culminating in the most ambitious SuperNova/Acceleration Probe (SNAP) [47] and Large Synoptic Survey Telescope (LSST) [48] surveys.

Furthermore, all future weak lensing surveys will provide photometric redshift information on the source galaxies. This additional information allows one to study tomographically the growth of the intervening large scale structure and the distanceredshift relation [49]. Indeed, weak lensing tomography is expected to reach a level of sensitivity comparable (and orthogonal) to SNIa for dark energy parameter determination (e.g., [50-56]).

The structure of this paper is as follows. We outline the principle of weak lensing tomography and present the formalism for computing the convergence power spectrum in section 2. In section 3, we discuss some of the systematic effects relevant for tomographic studies. The Fisher matrix formalism and the projected errors for various cosmological parameters are presented in section 4 . Section 5 contains our conclusions.

\section{Weak lensing tomography}

Distortions in the images of galaxies induced by density perturbations between the source and the observer can be described by the distortion tensor (e.g., [34,36]),

$$
\psi_{i j} \equiv \frac{\partial \theta_{s}^{i}}{\partial \theta^{j}}-\delta_{i j}=\left(\begin{array}{cc}
-\kappa-\gamma_{1} & -\gamma_{2} \\
-\gamma_{2} & -\kappa+\gamma_{1}
\end{array}\right),
$$

where $\boldsymbol{\theta}_{s}$ and $\boldsymbol{\theta}$ denote the angular position of a light ray in the source and the image planes respectively, and the tensor $\psi_{i j}$ decomposes into a convergence (i.e., magnification, $\kappa \ll 1$ ) and two shear (i.e., stretching, $\gamma_{1}, \gamma_{2} \ll 1$ ) components. In the Born approximation, the convergence component is given by a weighted projection of the density fluctuations $\delta \equiv \delta \rho / \rho$ along the line of sight,

$$
\kappa(\boldsymbol{\theta})=\int_{0}^{\chi_{h}} d \chi W(\chi) \delta(\chi, \chi \boldsymbol{\theta}),
$$

where $\chi=\int_{0}^{z} d z / H(z)$ is the comoving radial distance, $H(z)$ the Hubble parameter, and $\chi_{h}$ denotes the distance to the horizon. The assumption of a flat universe is implicit.

The lensing weighting function is defined as

$$
W(\chi)=\frac{W_{0}}{\bar{n}_{\text {gal }}} a^{-1}(\chi) \chi \int_{\chi}^{\chi_{h}} d \chi^{\prime} n_{\text {gal }}\left(\chi^{\prime}\right) \frac{\chi^{\prime}-\chi}{\chi^{\prime}},
$$

with $W_{0}=(3 / 2) \Omega_{m} H_{0}^{2}$, and $n_{\text {gal }}(\chi)=H(z) n_{\text {gal }}(z)$, where $n_{\text {gal }}(z)$ is the number of source galaxies between redshift $z$ and $z+d z$ per steradian, and satisfies the normalisation condition $\int_{0}^{\infty} d z n_{\text {gal }}(z)=\bar{n}_{\text {gal }}$. It is generally assumed that $n_{\text {gal }}(z)$ takes the form

$$
n_{\text {gal }}(z)=\bar{n}_{\text {gal }} \frac{\beta}{z_{0} \Gamma\left(\frac{1+\alpha}{\beta}\right)}\left(\frac{z}{z_{0}}\right)^{\alpha} \exp \left[-\left(\frac{z}{z_{0}}\right)^{\beta}\right]
$$


where the parameters $\alpha, \beta$ and $z_{0}$ are chosen to fit the observed galaxy redshift distribution. Here, we fix $\alpha=2$ and $\beta=2$. The choice of the parameter $z_{0}$ depends on the type of weak lensing survey: for a ground-based "wide" survey, $z_{0}=1$ corresponds to a median galaxy redshift $z_{\text {med }} \sim 1$; for a space-based "deep" survey, the choice of $z_{0}=1.5$ gives $z_{\text {med }} \sim 1.5$ (e.g., $[57,58]$ ).

Where photometric redshift information on the source galaxies is available and the sources separable into tomography bins, equations (2.2) and (2.3) can be generalised to give the convergence of the $i$ th bin via

$$
\kappa(\boldsymbol{\theta}) \rightarrow \kappa_{i}(\boldsymbol{\theta}), \quad W(\chi) \rightarrow W_{i}(\chi), \quad n_{\text {gal }}(z) \rightarrow n_{i}(z), \quad \bar{n}_{\text {gal }} \rightarrow \bar{n}_{i}
$$

where $\bar{n}_{i}=\int_{0}^{\infty} d z n_{i}(z)$ is the number density of source galaxies in the $i$ th tomography bin, and $\sum_{i} n_{i}(z)=n_{\text {gal }}(z)$ by construction. A simple model for $n_{i}(z)$ would be $n_{i}(z)=\Theta\left(z_{i+1}-z\right) \Theta\left(z-z_{i}\right) n_{\text {gal }}(z)$, where $z_{i}$ and $z_{i+1}$ denote the lower and upper limits of the $i$ th tomography bin.

\subsection{Lensing observables}

Since lensing by large scale density perturbations leads to image distortions typically of order $1 \%$ or less (much smaller than the intrinsic scatter in galaxy shapes), cosmological weak lensing must be measured statistically with a large ensemble of sources and lenses. By decomposing the convergence field into two-dimensional Fourier modes,

$$
\kappa_{i}(\boldsymbol{\theta})=\sum_{\boldsymbol{\ell}} \tilde{\kappa}_{\boldsymbol{\ell}}^{i} \exp (i \boldsymbol{\ell} \cdot \boldsymbol{\theta})
$$

one can define the angular power spectrum of the convergence at multipole $\ell$ between the $i$ th and $j$ th tomographic bin as

$$
\left\langle\tilde{\kappa}_{\ell}^{i} \tilde{\kappa}_{\ell^{\prime}}^{j *}\right\rangle=(2 \pi)^{2} \delta\left(\boldsymbol{\ell}-\boldsymbol{\ell}^{\prime}\right) C_{\ell}^{i j} .
$$

In the Limber approximation (where only modes with longitudinal wavenumbers $k_{3} \lesssim \chi^{-1}<\ell / \chi$ contribute to the correlation), the convergence power spectrum is related to the three-dimensional matter power spectrum $P_{m}(k)$ via $[59,60]$

$$
C_{\ell}^{i j}=\int_{0}^{\chi_{h}} d \chi W_{i}(\chi) W_{j}(\chi) \chi^{-2} P_{m}(k=\ell / \chi, z),
$$

assuming a flat universe. Thus weak lensing tomography with $n_{\mathrm{t}}$ bins yields $n_{\mathrm{t}}$ auto $(i=j)$ and $n_{\mathrm{t}}\left(n_{\mathrm{t}}-1\right) / 2$ cross $(i \neq j)$ correlation spectra. The case of $n_{\mathrm{t}}=1$ corresponds to lensing without tomography, and we shall be using these two terms interchangeably throughout the text.

Figure 1 shows an example of the convergence auto and cross power spectra expected for tomography with two bins. It is important to note that these power spectra are strongly correlated, and in their entirety do not provide as much information as a naïve interpretation might suggest. The gain in information from tomographic binning can be quantified by the correlation coefficient between the power spectra of the individual tomography bins, $R^{i j} \equiv C_{\ell}^{i j} / \sqrt{C_{\ell}^{i i} C_{\ell}^{j j}}$ [49]. A strong correlation (i.e., $R^{i j} \sim 1$ ) between two bins means that further subdivision is futile. 


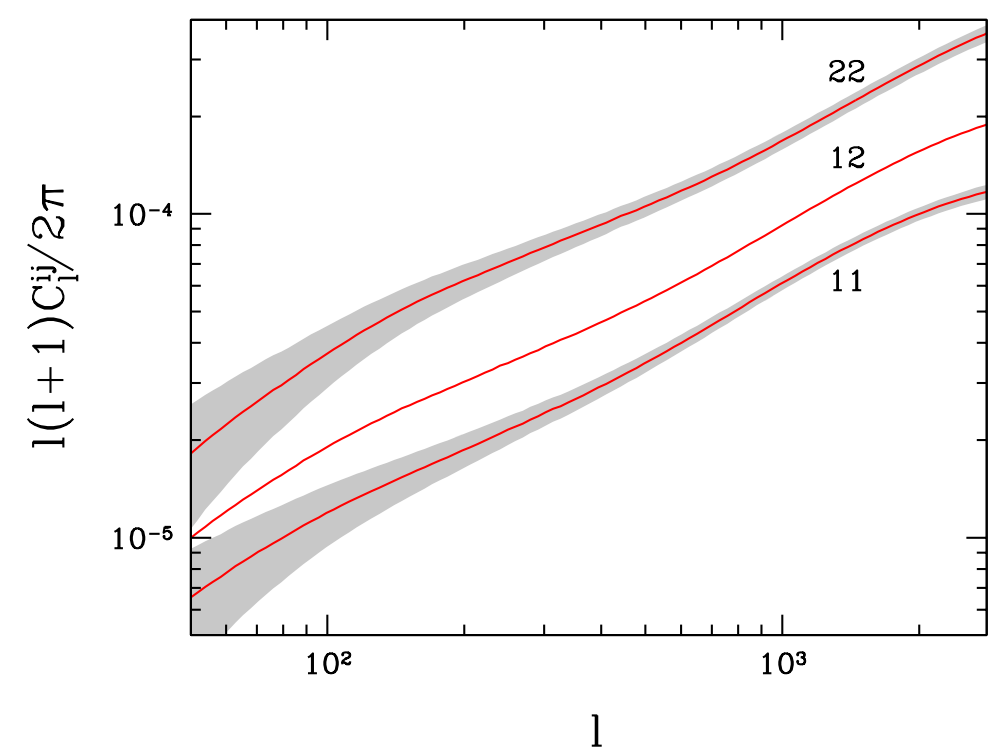

Figure 1. Convergence auto and cross power spectra for the standard $\Lambda$ CDM model, assuming the galaxy distribution function (2.4) and two tomography bins at $0 \leq z \leq 1.5$ and $1.5<z \leq 3$. The $1 \sigma$ error bands are computed for a lensing survey with sky coverage $f_{\text {sky }}=0.01, \bar{n}_{\text {gal }}=30 \operatorname{arcmin}^{-2}$, and $\gamma_{\mathrm{rms}}=0.4$, smeared over bands of width $\Delta \ell=\ell / 4$. See section 4 for definitions.

Note that while we have discussed only the convergence, existing and future weak lensing surveys will measure the source galaxies' ellipticities and hence the two shear components. However, by decomposing the shear signal into a gradient ("E-mode") and a curl ("B-mode") component, it can be shown that the statistics of the $E$-mode shear and of the convergence are identical (e.g., [61]). On the other hand, the $B$-mode is expected to yield a vanishing spectrum and therefore serves as a powerful test for residual systematics $[62,63]$. Henceforth, we shall consider the convergence power spectrum as representative of the lensing signatures.

\subsection{Dark energy and neutrinos}

The weak lensing signal (2.8) comprises of two distinct components: the lensing weights $W(\chi)$ and the matter power spectrum $P_{m}(k)$. While the latter quantifies the universal matter distribution, the role of the former, in broad terms, is to project this threedimensional distribution onto the two-dimensional sky for the observer. In general, for a single source at comoving distance $\chi_{0}$, the function $W(\chi)$ peaks at roughly $\chi_{0} / 2$. Thus, within the $\Lambda \mathrm{CDM}$ framework, a typical source distribution such as (2.4) peaking at $z \sim 1$ will be most efficiently lensed by structures at $z \sim 0.5$ (cf. Figure 2 ).

The effects of dark energy are manifest in both the lensing weights and the matter power spectrum. In the case of $W(\chi)$, the dark energy density $\Omega_{\mathrm{de}}$ and equation of state $w(z)$ determine the Hubble expansion rate at low redshifts,

$$
H^{2}(z)=H_{0}^{2}\left[\left(1-\Omega_{\mathrm{de}}\right)(1+z)^{3}+\Omega_{\mathrm{de}} e^{3 \times \int_{0}^{z} d z^{\prime}\left[1+w\left(z^{\prime}\right)\right]}\right],
$$


and hence the distance-redshift relation, $\chi=\int_{0}^{z} d z / H(z)$, responsible for the projection (cf. Figure 2). In the above equation we have assumed spatial flatness. This assumption will be kept throughout the analysis.

On the other hand, dark energy suppresses the growth of structure relative to the case of a flat, matter-dominated universe. In the absence of massive neutrinos, the linear matter power spectrum evolves as $P_{m}(k, a)=D^{2}(a) P_{m}(k, a=1)$, independently of $k$. Here, $D(a)$ is the growth function normalised to $D(a=1)=1$, and can be obtained by solving the differential equation (e.g., [64])

$$
2 \frac{d^{2} g}{d \ln a^{2}}+\left[5-3 w(a) \Omega_{\mathrm{de}}(a)\right] \frac{d g}{d \ln a}+3[1-w(a)] \Omega_{\mathrm{de}}(a) g(a)=0,
$$

where $g(a)=D(a) / a$, and $\Omega_{\mathrm{de}}(a)$ is the dark energy density at epoch $a$. The effects of $\Omega_{\mathrm{de}}$ and $w$ on the growth function are generally mild compared to those on $W(\chi)$. For the case of $w=-1.2$, for example, the lensing weight $W(\chi)$ increases by a uniform $3 \rightarrow 4 \%$ relative to the $w=-1$ case across $0<z<2$, while the change in the growth function $D(a)$ is a minute $-0.05 \%$ at $z=0.5$ at the peak of the lensing efficiency and only reaches $-2 \%$ at $z=2$ at the tail of $W(\chi)$. Thus the dominant constraints on the dark energy parameters will come from projection. In addition, $\Omega_{\mathrm{de}}\left(=1-\Omega_{m}\right)$ impacts immediately on the epoch of matter-radiation equality, which in turn determines the location of the turning point in the matter power spectrum.

Neutrino masses in the sub-eV range do not play a role in the universal expansion rate at low redshifts. Their early free-streaming effect on the shape of the matter power spectrum is however well known. As mentioned earlier, the loss of power at large wavenumbers due to a large $m_{\nu}$ can be offset by simultaneously translating the matter power spectrum towards large values of $k$. This translation can be accomplished by increasing (decreasing) $\Omega_{m}\left(\Omega_{\mathrm{de}}\right)$ and hence moving the matter-radiation equality epoch forward to an earlier time when the causal horizon is smaller. In this way, $m_{\nu}$ and $\Omega_{\mathrm{de}}$ form a degenerate pair.

Lensing without tomography, like angular galaxy surveys, makes use of a projected signal accumulated over a large distance $\left(\sim 4000 h^{-1} \mathrm{Mpc}\right)$. The information content of this signal, especially in the radial $\chi$ direction, is therefore limited. Tomography binning allows one to recover some radial information through a set of lensing weights $W_{i}(\chi)$ that peak at different values of $\chi$. This is particularly useful for establishing the distance-redshift relation and hence breaking the $\Omega_{\mathrm{de}}, w$ degeneracy. As shown in Figure 2, two sets of $\left\{\Omega_{\mathrm{de}}, w\right\}$ with lensing weights that are indistinguishable to the naked eye in the case of no tomography begin to show perceivable signs of differences when three tomography bins are used.

Tomography will not improve directly the neutrino mass bound. Even though massive neutrinos continue to affect the growth of small scale density fluctuations at low redshifts, the shape of the matter power spectrum evolves by a negligible amount over the $z$ range probed by weak lensing. To illustrate this point, we note that for $\sum m_{\nu}=0.3 \mathrm{eV}$, the normalised power spectrum $P_{m}(k) / P_{m}(k \rightarrow 0)$ changes by less than $2 \%$ at large values of $k$ between $z=0$ and $z=1.5$. This is smaller than the overall 


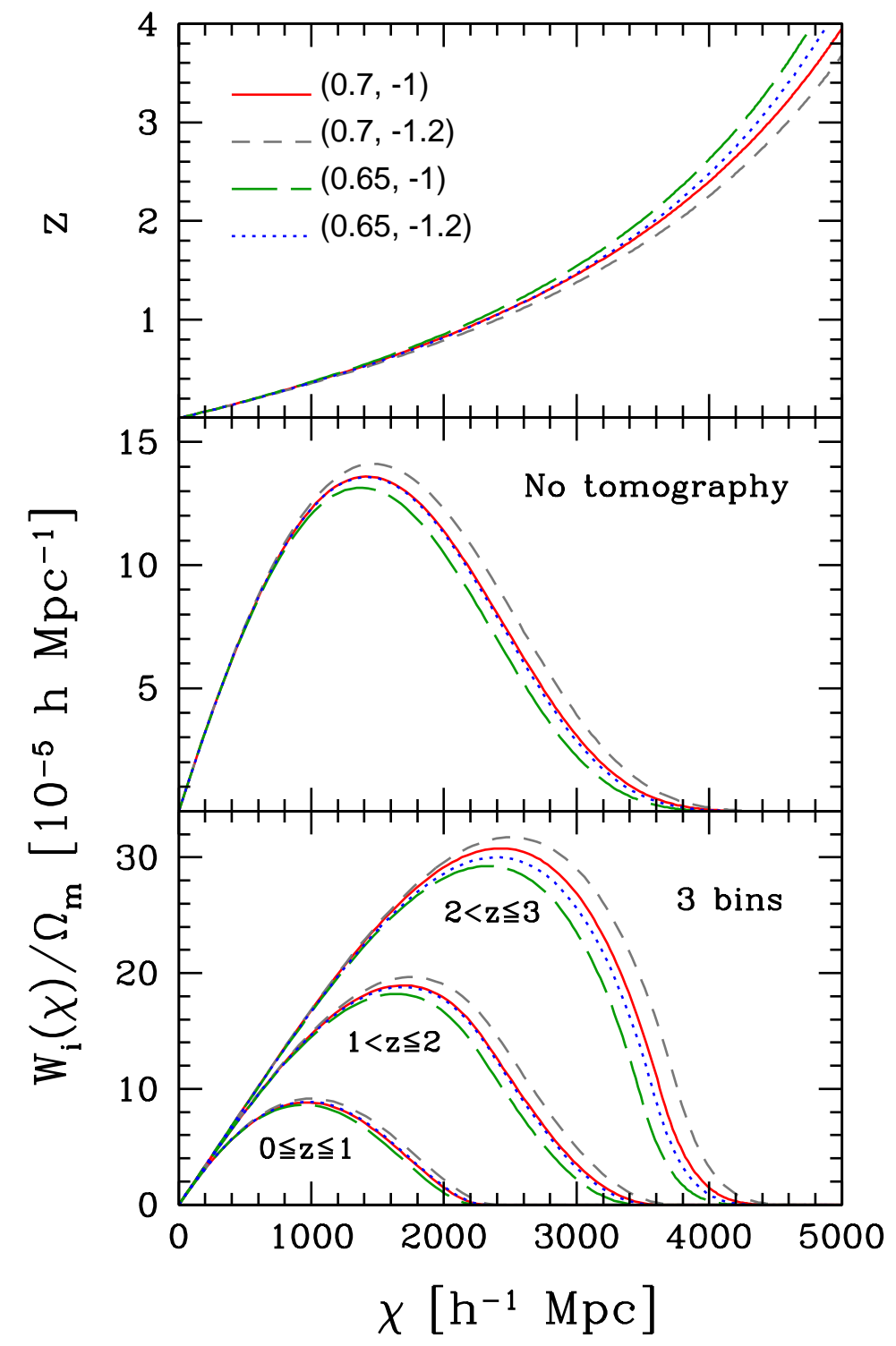

Figure 2. Top: Distance-redshift relation for four sets of dark energy parameters $\left\{\Omega_{\mathrm{de}}, w\right\}$ listed in the Figure, where $w$ is taken to be constant in time. Middle: Lensing weights $W(\chi) / \Omega_{m}$, without tomography, for the same four sets of $\left\{\Omega_{\mathrm{de}}, w\right\}$, assuming the galaxy distribution function (2.4) with $\alpha=2, \beta=2$, and $z_{0}=1$. Bottom: Lensing weights for three tomographic divisions at $0 \leq z \leq 1,1<z \leq 2$, and $2<z \leq 3$.

power suppression expected even for a $0.07 \mathrm{eV}$ neutrino today $\left(\sim 8 \Omega_{\nu} / \Omega_{m} \sim 5 \%\right)$. Nonetheless, once the $\Omega_{\mathrm{de}}, w$ degeneracy is lifted by projection effects, the $\Omega_{\mathrm{de}}, m_{\nu}$ shape degeneracy will be correspondingly reduced and the bound on $\sum m_{\nu}$ tightened.

\section{Systematic effects}

A number of systematic effects have been identified that could severely limit the potential of weak lensing surveys for cosmological parameter extraction. We discuss 
some of these below. For other systematics not covered here, we refer the reader to [36].

\subsection{Photometric redshift uncertainties}

Future surveys will observe a projected billion galaxies, a sample too large for individual spectroscopic redshift measurements. By observing the galaxy broad band features (i.e., broad band photometry) and comparing them to predictions from galaxy spectral energy distributions, redshift information can be obtained much more quickly at the cost of lower accuracy.

The uncertainties associated with the photometrically measured redshift are typically $\pm 0.034 \rightarrow 0.1$ (Hubble Deep Field [65], CFHTLS [42], SNAP with IRfilters [57], Combo-17 survey [66] with multi-colour filters etc.), compared with \pm 0.001 using spectroscopic methods (DEEP2 [67], VVDS [68], etc.). Assigning redshifts to galaxies using photometric methods should not contribute significantly to the error budget in weak lensing (without tomography) measurements. For tomographic studies, however, binning with photometric redshifts $z_{\mathrm{ph}}$ that suffer from uncontrolled scatter and bias relative to the true (spectroscopic) redshifts $z$ can lead to considerable error degradation in the dark energy parameter extraction. The authors of $[69,70]$ found that the scatter and the bias per redshift interval $\delta z=0.1$ need to be known to an accuracy of $0.003 \rightarrow 0.01$, in order not to degrade the errors in $\Omega_{\mathrm{de}}$ and $w$ by more than a factor of 1.5 .

We account for uncertainties in the photometric redshift following the approach of [69]. The true number of galaxy per steradian at redshift $[z, z+d z]$ in the $i$ th tomography bin is defined as

$$
n_{i}(z)=\int_{z_{\mathrm{ph}}^{i}}^{z_{\mathrm{ph}}^{i+1}} d z_{\mathrm{ph}} n_{\text {gal }}(z) p\left(z_{\mathrm{ph}} \mid z\right),
$$

where the function $p\left(z_{\mathrm{ph}} \mid z\right)$ is the probability that a given $z$ will be measured as $z_{\mathrm{ph}}$. This formulation assumes that $n_{\text {gal }}(z)$ will be accurately determined by an independent, dedicated spectroscopic survey (an alternative formulation for the case of a photometrically derived $n_{\text {gal }}(z)$ can be found in [70]). For simplicity, we choose $p\left(z_{\mathrm{ph}} \mid z\right)$ to be a Gaussian,

$$
p\left(z_{\mathrm{ph}} \mid z\right)=\frac{1}{\sqrt{2 \pi} \sigma_{z}} \exp \left[-\frac{\left(z_{\mathrm{ph}}+z_{\mathrm{bias}}-z\right)^{2}}{2 \sigma_{z}^{2}}\right],
$$

where $\sigma_{z}(z)$ and $z_{\text {bias }}(z)$ are the scatter and the bias parameters respectively. Following [69], we discretise $\sigma_{z}(z)$ and $z_{\text {bias }}(z)$ into $N_{\mathrm{pz}}=31$ sets of parameters $\left\{\sigma_{z}^{i}, z_{\text {bias }}^{i}\right\}$, where

$\sigma_{z}^{i} \equiv \sigma_{z}\left(z_{i}\right)$ and $z_{\text {bias }}^{i} \equiv z_{\text {bias }}\left(z_{i}\right)$, on an equally spaced grid from $z=0$ to $z=3$. Later on, each of $\sigma_{z}^{i}$ and $z_{\text {bias }}^{i}$ will be treated as a free parameter in our error forecast analysis.

The exact forms of $\sigma_{z}(z)$ and $z_{\text {bias }}(z)$ depend on how the photometric redshift is measured by the lensing survey. For example, $\sigma_{z}$ can be reduced by going to space (to eliminate the problem of absorption in the Earth's atmosphere), or by implementing IR-filters (sensitive to galaxies at $1 \leq z \leq 2$ ). Another possibility is to calibrate the survey internally by doing spectroscopy on a fraction of the source galaxies [71]. 
In this study, we choose as our fiducial model $\sigma_{z}^{i}=0.05$ and $z_{\text {bias }}^{i}=0$, and for these values, we have verified that the accuracy requirements for the scatter and the bias are roughly consistent with those found in [69].

\subsection{Shear measurement errors}

Technically, shear measurement is performed not on the actual galaxy images, but on images convolved with a point spread function (PSF) which describes the various image distortions originating from the telescope, the camera, and, in the case of ground-based surveys, the atmosphere. Errors in the lensing signal can therefore arise from uncorrected spatial and temporal variations in the PSF, as well as from the deconvolution and shear estimation method itself. Shear measurement errors can generally be discussed under the umbrellas of multiplicative and additive errors.

3.2.1. Multiplicative errors A number of algorithms have been developed so far to estimate the shear in an observed image, all of which must be calibrated against simulations (see, e.g., [72,73] for a summary). Uncertainties in the calibration can lead to the measured shear being over- or under-estimated by a constant multiplicative factor, and hence impact directly on the $\Omega_{m}$ and $\sigma_{8}$ parameter determination. Shear calibration errors are estimated to be $2 \%$ currently, and are expected to be controlled to $1 \%$ by the time SNAP and/or LSST start [74].

To incorporate this effect into our analysis, we follow the approach of [70], and define the theoretical convergence power spectrum as

$$
\hat{C}_{\ell}^{i j}=C_{\ell}^{i j} \times\left(1+f_{i}+f_{j}\right),
$$

where $f_{i}$ is the irreducible part of the shear calibration error in the $i$ th tomography bin, after averaging over all directions and redshifts within the bin. Thus, for tomography with $n_{\mathrm{t}}$ bins, there are $n_{\mathrm{t}}$ shear bias parameters.

3.2.2. Additive errors Additive errors arise when artificial shear signals are induced by, e.g., anisotropies in the PSF. In general, the PSF varies both spatially and temporally, and the anisotropies incurred by these variations must be calibrated, usually using stars in the observed fields, for each individual image. Current simulations suggest that additive PSF errors can be controlled to under $10^{-3}[74]$.

The effects of additive errors are more difficult to parameterise, since the errors will generally depend on $\ell$. A first attempt in [70] defines the total convergence power spectrum as $\hat{C}_{\ell}^{i j}=C_{\ell}^{i j}+C_{\ell}^{\text {add }}$, where the additive error spectrum $C_{\ell}^{\text {add }}$ is assumed to be a power law in $\ell$. Within this framework, the authors of [70] conclude that additive errors must be controlled to $\sim 10^{-5}$. We shall not be considering additive errors in this work, but simply note here that the size of current simulations are too small to determine if the $10^{-5}$ accuracy requirement is realistic [74]. 


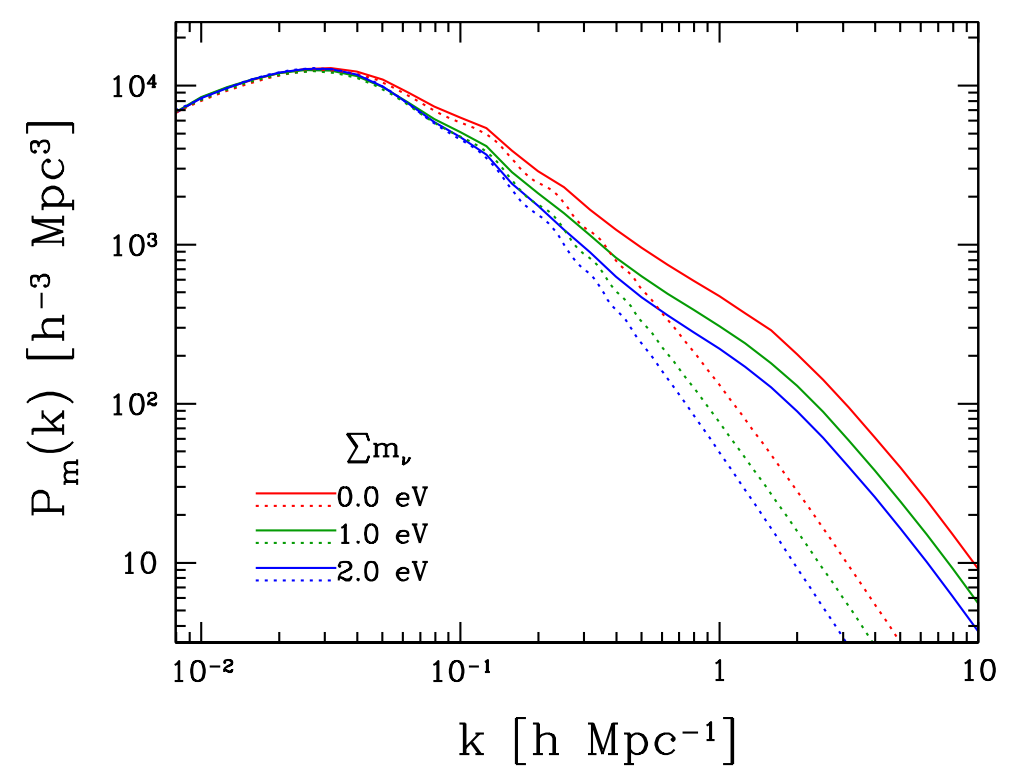

Figure 3. Nonlinear matter power spectra constructed from the halo model for various neutrino masses (solid lines). Also shown are the corresponding power spectra from the linear theory (dotted lines).

\subsection{Matter power spectrum}

The sensitivity of weak lensing surveys peaks at scales of several arcminutes, i.e., $\ell \sim 1000$ [75] (cf. Figure 1). At these scales the shear statistics is dominated by density perturbations of comoving wavenumbers $k \sim 1 \rightarrow 10 \mathrm{hMpc}^{-1}$ at $z \sim 0.5$. Thus, weak lensing probes a convolution of linear and nonlinear scales at present and earlier times, and the linear power spectra $P^{\mathrm{L}}(k)$ computed by, e.g., CMBFAST [76] alone will not suffice to determine total matter power spectrum $P_{m}(k)$.

Assuming that baryons trace CDM exactly, accurate predictions of $P_{m}(k)$ can be obtained by means of collisionless $N$-body simulations. At present various cosmological simulation codes agree at the $5 \rightarrow 10 \%$ level [77] for $\Lambda \mathrm{CDM}$ cosmologies. In the future, however, $P_{m}(k)$ will have to be calibrated to about $1 \rightarrow 2 \%$ accuracy in order not to compromise the cosmological parameter extraction from ambitious surveys such as SNAP and LSST [78]. Inclusion of baryon physics introduces an extra layer of complexity; dissipative processes such as radiative cooling and star formation enters at

$k \gtrsim 1 h \mathrm{Mpc}$, and require in addition the use of hydrodynamical simulations to accurately model the behaviour of the baryon gas. We note in passing that large volume simulations of dark energy cosmologies to date have focussed mainly on $\Lambda \mathrm{CDM}$, although there have been some efforts in the direction of $w \neq-1$ and/or a time-varying equation of state (e.g., [79-82]). To our knowledge, simulations of dark energy cosmologies with mixed dark matter have not yet been reported in the literature.

A number of semi-analytical methods and fitting formulae have been devised [8387], some of which tested to roughly $10 \%$ accuracy against $N$-body simulations $[53,88]$, to map between the linear and nonlinear power spectra in CDM cosmologies. Virtually 
all weak lensing parameter constraint analyses to date employ one of these mapping methods. In this paper, we correct for nonlinearity using the halo model [84-86], assuming that it can be generalised to general dark energy and mixed dark matter cosmologies. Here, the total matter power spectrum is taken to be a weighted average of the neutrino $(\nu)$, and the combined baryon $(b)$ and CDM $(c)$ power spectra,

$$
P_{m}(k)=\left[f_{\nu} \sqrt{P_{\nu}^{\mathrm{L}}(k)}+\left(f_{b}+f_{c}\right) \sqrt{P_{b+c}^{\mathrm{NL}}(k)}\right]^{2},
$$

with weights $f_{i}=\Omega_{i} / \Omega_{m}, i=\nu, b, c$, and $\Omega_{m} \equiv \Omega_{\nu}+\Omega_{b}+\Omega_{c}$. The nonlinear baryon and CDM power spectrum $P_{b+c}^{\mathrm{NL}}(k)$ is mapped from its linear counterpart,

$$
P_{b+c}^{\mathrm{L}}(k)=\left(f_{b}+f_{c}\right)^{-1}\left[f_{b} \sqrt{P_{b}^{\mathrm{L}}(k)}+f_{c} \sqrt{P_{c}^{\mathrm{L}}(k)}\right]^{2}
$$

following the halo model prescription. See Appendix A for details.

Figure 3 shows the nonlinear matter power spectra constructed in this fashion for various neutrino masses. Clearly, the suppression of power due to neutrino freestreaming extends well into the nonlinear regime $(k \gtrsim 0.2 \mathrm{hMpc})$. Indeed, the halo model predicts an even stronger suppression on nonlinear scales than in the linear region $(k \lesssim 0.2 \mathrm{hMpc}$ ). However, compared with their corresponding uncorrected power spectra (i.e., power spectra calculated from the linear theory, extended into the nonlinear regime), this relative suppression, i.e., the ratio $\Delta P_{m}(k) / P_{m}(k)$, in the corrected spectra seems to be somewhat weaker. (Observe in Figure 3 that the gaps between the solid lines seem to be narrower than those between the dotted lines at a given $k$.) This result remains to be verified by simulations.

We do not consider nonlinearity in the neutrino power spectrum $P_{\nu}^{\mathrm{L}}(k)$ incurred from late-time infall into existing halos [89], since it affects the total matter power spectrum and hence the weak lensing observables only at the $\sim 0.1 \%$ level $[90,91]$. We ignore also corrections due to cooling baryons and the intra-cluster gas [92,93], although results from recent $N$-body/hydrodynamical simulations suggest that baryon

physics affects the weak lensing signal at the $\lesssim 0.5 \%$ and the $1 \rightarrow 10 \%$ levels at $\ell<1000$ and $1000<\ell<10000$ respectively [94].

\section{Error forecast}

We use the Fisher matrix formalism to estimate the constraints on various cosmological parameters from weak lensing tomography.

\subsection{Fisher matrix}

The Fisher matrix is defined as

$$
F_{\alpha \beta}=-\left\langle\frac{\partial^{2} \ln L}{\partial p_{\alpha} \partial p_{\beta}}\right\rangle,
$$

where $L(\boldsymbol{x}, \boldsymbol{p})$ is the likelihood function of the data set $\boldsymbol{x}$ given the parameters $\boldsymbol{p}=\left\{p_{1}, \ldots, p_{\alpha}\right\}$ of the theoretical model. When evaluated in the vicinity of the 
Table 1. Generic weak lensing survey parameters.

\begin{tabular}{lcccc}
\hline & $f_{\text {sky }}$ & $z_{0}$ & $\gamma_{\text {rms }}$ & $\bar{n}_{\text {gal }}\left(\operatorname{arcmin}^{-2}\right)$ \\
\hline Wide, LSST-like & 0.7 & 1.0 & 0.4 & 30 \\
Deep, SNAP-like & 0.01 & 1.5 & 0.25 & 100 \\
\hline
\end{tabular}

fiducial model, the Fisher matrix quantifies the best statistical error on the parameter $p_{\alpha}$ achievable with a given data set via

$$
\sigma\left(p_{\alpha}\right) \geq \sqrt{\left(\boldsymbol{F}^{-1}\right)_{\alpha \alpha}},
$$

where $\sigma\left(p_{\alpha}\right)$ denotes the $1 \sigma$ error on $p_{\alpha}$ after marginalisation over all other parameters.

Neglecting non-Gaussian corrections, the Fisher matrix for weak lensing tomography has the form

$$
F_{\alpha \beta}=\sum_{\ell_{\min }}^{\ell_{\max }}(\ell+1 / 2) f_{\mathrm{sky}} \Delta \ell \operatorname{Tr}\left(\widetilde{\boldsymbol{C}}_{\ell}^{-1} \frac{\partial \boldsymbol{C}_{\ell}}{\partial p_{\alpha}} \widetilde{\boldsymbol{C}}_{\ell}^{-1} \frac{\partial \boldsymbol{C}_{\ell}}{\partial p_{\beta}}\right) .
$$

Here, we take the lensing observable $\boldsymbol{C}_{\ell}$ to be the number weighted convergence power spectrum $\boldsymbol{C}_{\ell} \doteq \bar{n}_{i} \bar{n}_{j} \hat{C}_{\ell}^{i j}$, since it contains more information than does the binned spectrum $\hat{C}_{\ell}^{i j}$ to reflect changes in the photometric redshift parameters [69]. The variance $\widetilde{\boldsymbol{C}}_{\ell}$ is correspondingly defined as a number weighted sum of the convergence and the noise power spectra, $\widetilde{\boldsymbol{C}}_{\ell} \doteq \bar{n}_{i} \bar{n}_{j}\left(\hat{C}_{\ell}^{i j}+\delta_{i j} \gamma_{\mathrm{rms}}^{2} / \bar{n}_{i}\right)$. Note that in the case of fixed photometric redshift parameters, these definitions lead to the same $F_{\alpha \beta}$ as their more common representations, $\boldsymbol{C}_{\ell} \doteq \hat{C}_{\ell}^{i j}$ and $\widetilde{\boldsymbol{C}}_{\ell} \doteq \hat{C}_{\ell}^{i j}+\delta_{i j} \gamma_{\mathrm{rms}}^{2} / \bar{n}_{i}$. We consider a variety of tomography bin numbers $\left(n_{\mathrm{t}}=1,3,5,8\right)$, each with equal redshift binning $\Delta z$ between $z=0$ and $z=3$ (e.g., $\Delta z=0.6$ for $n_{\mathrm{t}}=5$ ).

The minimum and maximum multipoles $\ell_{\min }$ and $\ell_{\max }$ are taken to be 50 and 3000 respectively, the latter so as to avoid the highly non-Gaussian signals at the very small scales. Between $\ell_{\min }$ and $\ell_{\max }$ we use 100 logarithmic bins of width $\Delta \ell$; variations in the $\ell$ binning have little effect on our results, since the convergence power spectrum is fairly featureless. The other parameters - the sky coverage $f_{\text {sky }}$, the surface density of galaxies $\bar{n}_{\text {gal }}=\sum_{i} \bar{n}_{i}$, and their rms intrinsic ellipticity $\gamma_{\mathrm{rms}}$-are generally survey-dependent. In our analysis, we consider two generic weak lensing surveys: a ground-based LSST-like "wide" survey, and a space-based SNAP-like "deep" survey, with survey parameters displayed in Table 1.

We are primarily interested in how parameter constraints derived from CMB data can be improved with the inclusion of weak lensing. Since the primary CMB anisotropies and the galaxy shears probed by weak lensing surveys are generated at two vastly different epochs, we may take them to be uncorrelated. Thus the total Fisher matrix is simply the sum of two independent matrices,

$$
F_{\alpha \beta}^{\text {total }}=F_{\alpha \beta}^{\mathrm{WL}}+F_{\alpha \beta}^{\mathrm{CMB}} \text {, }
$$

which is a good approximation provided we confine the analysis to the unlensed CMB anisotropies. (Weak lensing of the $\mathrm{CMB}$ and of galaxies are necessarily strongly 
Measuring neutrino masses and dark energy with weak lensing tomography

Table 2. Experimental specifications for Planck, assuming one year of observation.

\begin{tabular}{ccccc}
\hline$f_{\text {sky }}$ & $\nu(\mathrm{GHz})$ & $\theta_{b}(\operatorname{arcmin})$ & $\Delta_{T}\left(10^{-6} T\right)$ & $\Delta_{E}\left(10^{-6} T\right)$ \\
\hline 0.65 & 100 & 9.5 & 2.5 & 4.0 \\
& 143 & 7.1 & 2.2 & 4.2 \\
& 217 & 5.0 & 4.8 & 9.8 \\
\hline
\end{tabular}

correlated since the lensing signals are generated by much of the same intervening large scale structure.)

The Fisher matrix for the unlensed $\mathrm{CMB}$ anisotropies $F_{\alpha \beta}^{\mathrm{CMB}}$ is given likewise by equation (4.3), but with $\widetilde{\boldsymbol{C}}_{\ell}=\boldsymbol{C}_{\ell}+\boldsymbol{N}_{\ell}$, and

$$
\boldsymbol{C}_{\ell}=\left(\begin{array}{cc}
C_{\ell}^{T T} & C_{\ell}^{T E} \\
C_{\ell}^{T E} & C_{\ell}^{E E}
\end{array}\right), \quad \boldsymbol{N}_{\ell}=\left(\begin{array}{cc}
N_{\ell}^{T T} & 0 \\
0 & N_{\ell}^{E E}
\end{array}\right),
$$

where $C_{\ell}^{T T}, C_{\ell}^{E E}$, and $C_{\ell}^{T E}$ are the auto and cross power spectra of the CMB temperature and $E$-type polarisation. These are computed using CMBFAST version 4.5.1 [76]. We do not consider $B$-type polarisation, although it will most likely be measured by the next generation of $\mathrm{CMB}$ probes. The noise power spectra $\boldsymbol{N}_{\ell}$ vary with the detector performance according to

$$
N_{\ell}^{a a}=\left[\sum_{\nu}\left(N_{\ell, \nu}^{a a}\right)^{-1}\right]^{-1}, \quad N_{\ell, \nu}^{a a}=\left(\theta_{b} \Delta_{a}\right)^{2} \exp \left[\ell(\ell+1) \theta_{b}^{2} / 8 \ln 2\right],
$$

where $\nu$ denotes the detector frequency channel, $\theta_{b}$ is the beam width, and $\Delta_{a}$ is the temperature/polarisation sensitivity per pixel. Table 2 shows the expected performance of the Planck satellite [95] (assuming one year of observation), and we take $\ell_{\max }=1500$ in our analysis.

Prior information on the photometric redshift uncertainties (i.e., the parameters $z_{\text {bias }}^{i}$ and $\sigma_{z}^{i}$ ) and the shear calibration bias (i.e., $f_{i}$ ) can be included in the analysis by adding the diagonal matrix $F_{\alpha \beta}^{\text {prior }}=\delta_{\alpha \beta} \sigma\left(p_{\alpha}\right)^{-2}$ to the total Fisher matrix (4.4).

\subsection{The fiducial model}

We consider a spatially flat cosmological model with eleven parameters, the fiducial values of which are as follows: $\sum m_{\nu}=0.07 \mathrm{eV}, \Omega_{\mathrm{de}}=0.7, \Omega_{b} h^{2}=0.0245$, $\Omega_{c} h^{2}=0.1225, w_{0}=-1, w_{a}=0, \tau=0.05, n_{s}=1, \alpha_{s}=0, \sigma_{8}=0.9$, and $N_{\text {eff }}=3$. In this model, the time-dependent dark energy equation of state is parameterised as $[96,97]$

$$
w(z)=w_{0}+w_{a}[1-a(z)] .
$$

See, e.g., [24-26] for alternative parameterisations. We also allow for nonstandard relativistic degrees of freedom $N_{\text {eff }} \neq 3$, and a running scalar spectral index $\alpha_{s}$ evaluated at $k_{0}=0.05 \mathrm{hMpc}^{-1}$. These extra parameters, $\left\{N_{\mathrm{eff}}, \alpha_{s}, w_{0}, w_{a}\right\}$, are known to be degenerate with the neutrino mass $m_{\nu}$. For simplicity, we take the number of massive neutrino species to be one. Spatial flatness implies $\Omega_{\mathrm{de}}+\Omega_{\nu}+\Omega_{b}+\Omega_{c}=1$. 


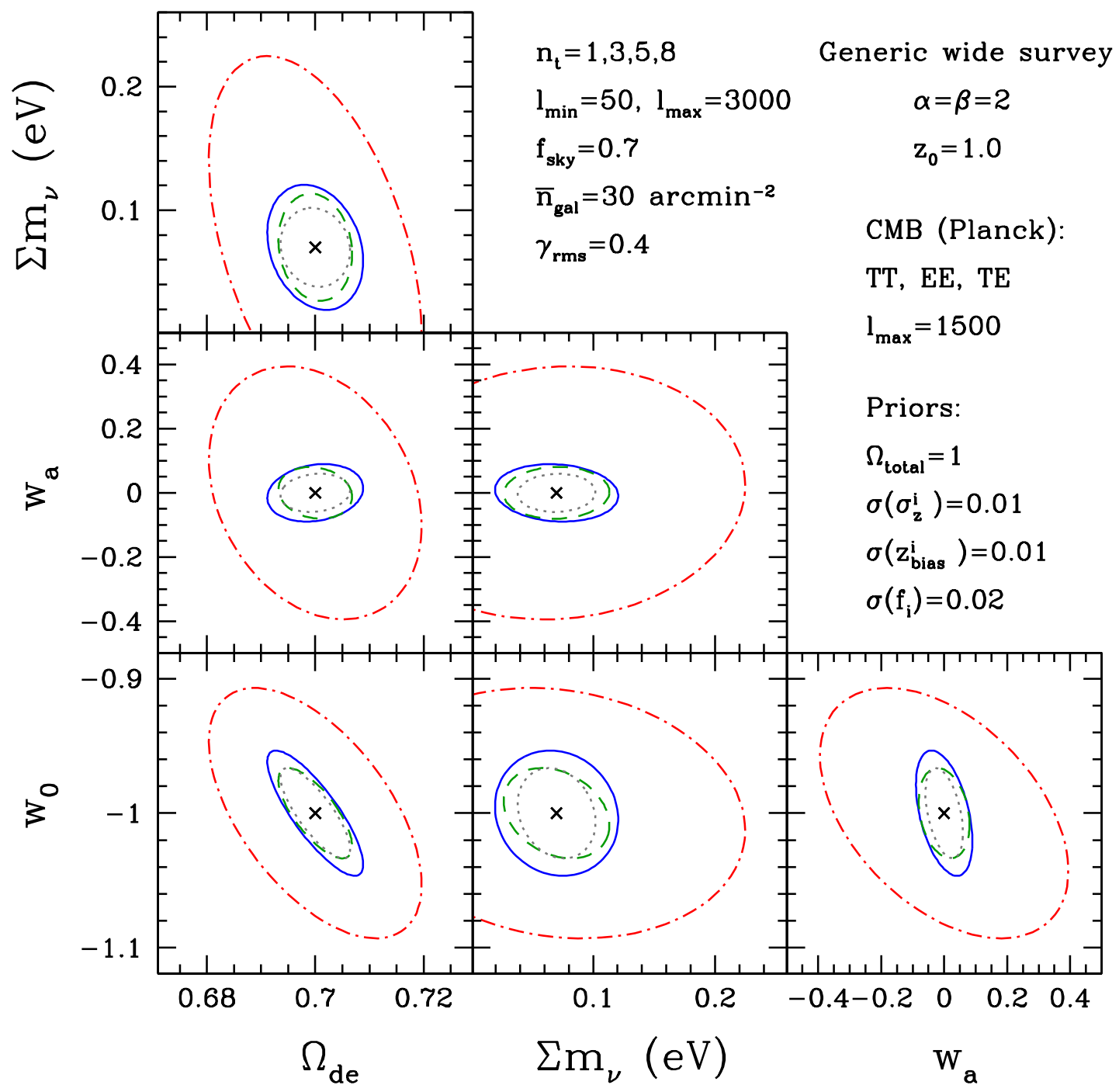

Figure 4. Projected $1 \sigma$ contours in the dark energy and neutrino parameter space from an LSST-like ground-based wide lensing survey (with and without tomography) and Planck for an eleven parameter cosmological model. The red (dot-dash), blue (solid), green (dashed), and gray (dotted) lines correspond to one, three, five, and eight tomography bins respectively. Each contour comes from marginalising over the other nine cosmological parameters and all $2 \times N_{\mathrm{pz}}+n_{\mathrm{t}}$ systematic parameters, with Gaussian priors imposed on the latter.

In addition to the eleven cosmological parameters, we marginalise over $2 \times N_{\mathrm{pz}}+n_{\mathrm{t}}$ nuisance parameters, $\sigma_{z}^{i}, z_{\text {bias }}^{i}$, and $f_{i}$. The fiducial values of these parameters are taken to be $\sigma_{z}^{i}=0.05, z_{\text {bias }}^{i}=0$, and $f_{i}=0$, and the Gaussian priors $\sigma\left(\sigma_{z}^{i}\right)=0.01$, $\sigma\left(z_{\text {bias }}^{i}\right)=0.01$, and $\sigma\left(f_{i}\right)=0.02$ are used in the marginalisation. We do not employ priors on the cosmological parameters. 


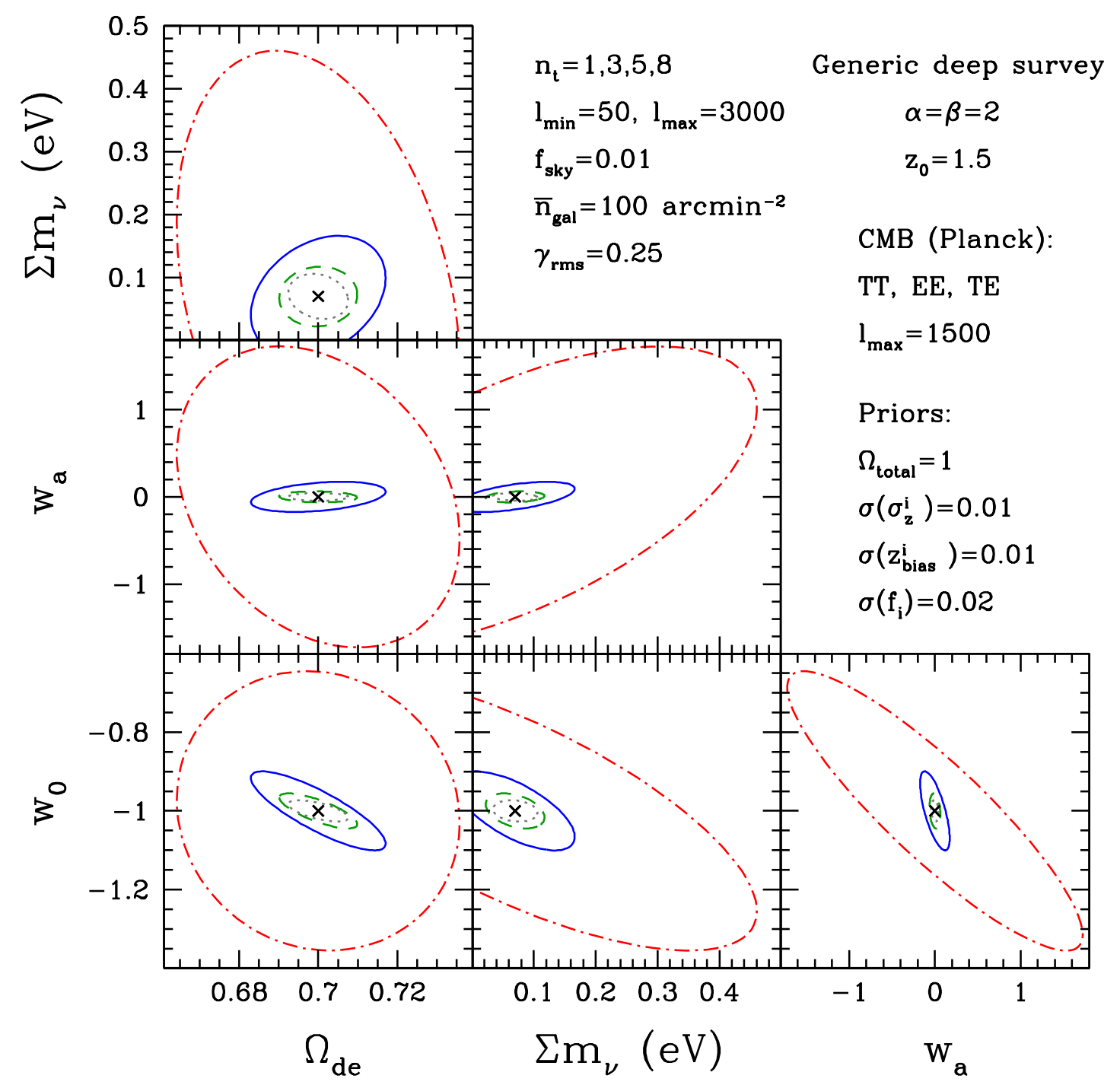

Figure 5. Same as Figure 4, but from a SNAP-like space-based deep survey and Planck.

\subsection{Results}

Figures 4 and 5 show the projected $1 \sigma$ contours in the dark energy and neutrino parameter space expected from two generic weak lensing surveys, with and without tomography, in combination with prospective CMB data from the Planck mission. (See Table 3 for all projected errors, and Table 4 for the parameter error correlation matrix $F_{\alpha \beta} / \sqrt{F_{\alpha \alpha} F_{\beta \beta}}$. $)$ Clearly, the addition of tomographic binning significantly reduces the errors for all four parameters, $\left\{\sum m_{\nu}, w_{0}, w_{a}, \Omega_{\mathrm{de}}\right\}$. Focussing on the LSST-like wide survey (Figure 4), we see that the improvement on the $w_{a}$ error estimate is roughly a factor of five. For the neutrino mass, the gain is slightly more moderate: roughly a factor of three difference between the cases of no tomography and tomography with five bins. This follows from the fact that tomography does not directly add extra information on 
Table 3. Projected $1 \sigma$ errors from various combinations of Planck and weak lensing. The labels "Wide" and "Deep" denote the two generic lensing surveys considered in this work, and the suffix number indicates the number of tomography bins used in the analysis.

\begin{tabular}{lccccc}
\hline & Planck & +Wide-1 & +Wide-5 & +Deep-1 & +Deep-5 \\
\hline$\sigma\left(\sum m_{\nu}\right)(\mathrm{eV})$ & 0.48 & 0.15 & 0.043 & 0.39 & 0.047 \\
$\sigma\left(\Omega_{\mathrm{de}}\right)$ & 0.08 & 0.020 & 0.0068 & 0.036 & 0.0099 \\
$\sigma\left(\Omega_{b} h^{2}\right)$ & 0.00028 & 0.00016 & 0.00013 & 0.00024 & 0.00014 \\
$\sigma\left(\Omega_{c} h^{2}\right)$ & 0.0026 & 0.0017 & 0.0015 & 0.0019 & 0.0015 \\
$\sigma\left(w_{0}\right)$ & 0.83 & 0.093 & 0.034 & 0.35 & 0.045 \\
$\sigma\left(w_{a}\right)$ & 4.0 & 0.39 & 0.081 & 1.7 & 0.063 \\
$\sigma(\tau)$ & 0.0046 & 0.0043 & 0.0042 & 0.0045 & 0.0043 \\
$\sigma\left(n_{s}\right)$ & 0.0089 & 0.0056 & 0.0028 & 0.0074 & 0.0047 \\
$\sigma\left(\alpha_{s}\right)$ & 0.024 & 0.013 & 0.0061 & 0.020 & 0.012 \\
$\sigma\left(\sigma_{8}\right)$ & 0.084 & 0.019 & 0.0076 & 0.030 & 0.0092 \\
$\sigma\left(N_{\mathrm{eff}}\right)$ & 0.19 & 0.11 & 0.067 & 0.14 & 0.093 \\
\hline
\end{tabular}

the shape of the matter power spectrum (see discussion in section 2.2).

The SNAP-like deep survey (Figure 5) offers even more significant relative improvement, since it probes further back in redshift where the effect of dark energy on the distance-redshift relation is more prominent (cf. Figure 2). However, owing to its limited sky coverage, the absolute errors (with tomography) are comparable to those from the wide survey. Should the value of $f_{\text {sky }}$ be increased, one can scale the errors presented here roughly via

$$
\sigma\left(p_{\alpha}, f_{\text {sky }}\right) \sim\left(f_{\text {sky }, \text { fid }} / f_{\text {sky }}\right)^{1 / 2} \sigma\left(p_{\alpha}, f_{\text {sky,fid }}\right),
$$

where $f_{\text {sky,fid }}$ denotes the sky coverage of the fiducial survey considered in this work. This expression is applicable for $\left\{\sum m_{\nu}, w_{0}, w_{a}, \Omega_{\mathrm{de}}\right\}$ with tomography, since the constraints on these parameters come predominantly from lensing, not from CMB.

It is interesting to compare these constraints with those derived from CMB alone. Table 3 shows the projected errors on various cosmological parameters from Planck alone, and in combination with a selection of lensing surveys. CMB alone has very little sensitivity to the dark energy equation of state; lensing is crucial for the determination of $w_{0}$ and $w_{a}$. On the other hand, although the projected error on the neutrino mass from Planck alone is comparable to what can be achieved today with $\mathrm{CMB}+\mathrm{LSS}+\mathrm{SNIa}+\mathrm{BAO}$, weak lensing (without tomography) brings it down to a level comparable to the projected sensitivities of future galaxy surveys (e.g., [98]). The addition of tomography binning pushes the error down even further.

Of course, these impressive sensitivities come at a price: appropriate priors have been imposed on the systematics parameters in order not to degrade the sensitivity to any one (cosmological) parameter by more than $\sim 50 \%$ relative to the best achievable error by a systematics-free lensing survey. Figure 6 shows the degradation in the projected sensitivities to $\left\{\sum m_{\nu}, w_{0}, w_{a}, \Omega_{\mathrm{de}}\right\}$ as a function of priors on the shear 


\begin{tabular}{|c|c|c|c|c|c|c|c|c|c|c|c|c|c|c|c|c|}
\hline (a) & $\sum m_{\nu}$ & $\Omega_{\mathrm{de}}$ & $\Omega_{b} h^{2}$ & $\Omega_{c} h^{2}$ & $w_{0}$ & $w_{a}$ & $\tau$ & $n_{s}$ & $\alpha_{s}$ & $\sigma_{8}$ & $N_{\text {eff }}$ & $f_{1}$ & $f_{2}$ & $f_{3}$ & $f_{4}$ & $f_{5}$ \\
\hline$\sum m_{\nu}$ & 1 & -0.13 & 0.038 & -0.077 & -0.27 & 0.040 & -0.016 & 0.19 & 0.30 & -0.093 & 0.068 & -0.064 & -0.22 & -0.24 & -0.13 & -0.038 \\
\hline$\Omega_{\mathrm{de}}$ & -0.45 & 1 & -0.14 & -0.16 & -0.73 & -0.15 & 0.056 & -0.36 & -0.21 & 0.79 & -0.32 & -0.011 & 0.041 & 0.042 & -0.17 & -0.053 \\
\hline$\Omega_{b} h^{2}$ & 0.17 & -0.51 & 1 & -0.0071 & 0.30 & -0.0026 & 0.076 & 0.18 & 0.43 & -0.22 & 0.18 & 0.047 & 0.19 & 0.21 & 0.14 & 0.049 \\
\hline$\Omega_{c} h^{2}$ & 0.035 & -0.35 & 0.096 & 1 & -0.023 & -0.031 & 0.0039 & 0.047 & -0.14 & -0.22 & 0.83 & 0.030 & -0.035 & -0.11 & 0.16 & 0.0084 \\
\hline$w_{0}$ & -0.14 & -0.57 & 0.56 & 0.14 & 1 & -0.25 & 0.0060 & 0.44 & 0.39 & -0.68 & 0.29 & 0.053 & 0.21 & 0.23 & 0.23 & 0.071 \\
\hline$w_{a}$ & 0.064 & -0.26 & -0.074 & 0.14 & -0.46 & 1 & 0.0017 & -0.047 & 0.090 & -0.11 & -0.030 & 0.051 & 0.022 & 0.038 & 0.18 & 0.068 \\
\hline$\tau$ & -0.081 & 0.13 & 0.021 & -0.036 & -0.016 & -0.065 & 1 & 0.14 & -0.16 & 0.059 & 0.052 & -0.0017 & 0.029 & 0.038 & 0.0055 & 0.00056 \\
\hline$n_{s}$ & 0.44 & -0.82 & 0.57 & 0.26 & 0.63 & 0.030 & -0.013 & 1 & 0.64 & -0.56 & 0.39 & 0.098 & 0.26 & 0.21 & 0.23 & 0.084 \\
\hline$\alpha_{s}$ & 0.60 & -0.80 & 0.59 & 0.15 & 0.38 & 0.27 & -0.17 & 0.85 & 1 & -0.29 & 0.37 & 0.058 & 0.15 & 0.16 & 0.18 & 0.058 \\
\hline$\sigma_{8}$ & -0.31 & 0.92 & -0.57 & -0.36 & -0.62 & -0.23 & 0.11 & -0.85 & -0.73 & 1 & -0.46 & -0.12 & -0.39 & -0.37 & -0.35 & -0.13 \\
\hline$N_{\text {eff }}$ & 0.37 & -0.77 & 0.50 & 0.72 & 0.52 & 0.090 & -0.047 & 0.79 & 0.73 & -0.79 & 1 & 0.074 & 0.15 & 0.088 & 0.16 & 0.057 \\
\hline$f_{1}$ & -0.68 & 0.14 & 0.16 & -0.04 & 0.23 & 0.12 & 0.068 & -0.019 & -0.19 & -0.20 & -0.038 & 1 & -0.013 & 0.16 & 0.049 & 0.026 \\
\hline$f_{2}$ & - & - & - & - & - & - & - & - & - & - & - & - & 1 & 0.23 & 0.24 & 0.10 \\
\hline$f_{3}$ & - & - & - & - & - & - & - & - & - & - & - & - & - & 1 & 0.098 & 0.13 \\
\hline$f_{4}$ & - & - & - & - & - & - & - & - & - & - & - & - & - & - & 1 & -0.0086 \\
\hline \multirow[t]{2}{*}{$f_{5}$} & - & - & - & - & - & - & - & - & - & - & - & - & - & - & - & 1 \\
\hline & & & & & & & & & & & & & & & & \\
\hline (b) & $\sum m_{\nu}$ & $\Omega_{\mathrm{de}}$ & $\Omega_{b} h^{2}$ & $\Omega_{c} h^{2}$ & $w_{0}$ & $w_{a}$ & $\tau$ & $n_{s}$ & $\alpha_{s}$ & $\overline{\sigma_{8}}$ & $N_{\text {eff }}$ & $\overline{f_{1}}$ & $\overline{f_{2}}$ & $f_{3}$ & $f_{4}$ & $\overline{f_{5}}$ \\
\hline$\overline{\sum m_{\nu}}$ & 1 & 0.048 & 0.057 & 0.039 & -0.37 & 0.19 & -0.012 & 0.14 & 0.17 & -0.11 & 0.15 & -0.0028 & 0.00015 & -0.072 & -0.016 & -0.034 \\
\hline$\Omega_{\mathrm{de}}$ & -0.30 & 1 & 0.0060 & -0.19 & -0.76 & -0.095 & 0.0084 & 0.0096 & 0.14 & 0.77 & -0.098 & 0.035 & 0.10 & 0.090 & -0.017 & -0.082 \\
\hline$\Omega_{b} h^{2}$ & -0.46 & -0.075 & 1 & 0.060 & 0.30 & 0.020 & 0.032 & 0.44 & 0.58 & -0.088 & 0.40 & 0.014 & 0.11 & 0.13 & 0.12 & 0.070 \\
\hline$\Omega_{c} h^{2}$ & -0.0055 & -0.18 & 0.071 & 1 & 0.013 & 0.32 & -0.029 & 0.25 & 0.14 & -0.27 & 0.75 & -0.0021 & -0.026 & -0.057 & -0.0038 & 0.020 \\
\hline$w_{0}$ & -0.72 & -0.080 & 0.76 & -0.036 & 1 & -0.20 & 0.0026 & 0.33 & 0.28 & -0.57 & 0.27 & -0.022 & -0.027 & 0.034 & 0.079 & 0.094 \\
\hline$w_{a}$ & 0.60 & -0.28 & -0.66 & 0.15 & -0.89 & 1 & -0.016 & 0.11 & 0.12 & -0.16 & 0.27 & 0.027 & 0.12 & 0.045 & 0.040 & 0.097 \\
\hline$\tau$ & -0.23 & 0.15 & 0.19 & -0.086 & 0.26 & -0.30 & 1 & 0.012 & -0.15 & 0.036 & -0.026 & 0.00011 & 0.0054 & 0.014 & 0.012 & 0.0065 \\
\hline$n_{s}$ & -0.17 & -0.25 & 0.71 & 0.30 & 0.55 & -0.42 & 0.083 & 1 & 0.87 & -0.18 & 0.73 & 0.017 & 0.12 & 0.12 & 0.10 & 0.061 \\
\hline$\alpha_{s}$ & 0.34 & -0.32 & 0.030 & 0.33 & -0.38 & 0.57 & -0.29 & 0.41 & 1 & 0.065 & 0.72 & 0.011 & 0.059 & 0.023 & -0.018 & -0.030 \\
\hline$\sigma_{8}$ & -0.44 & 0.87 & -0.17 & -0.17 & -0.093 & -0.13 & 0.12 & -0.37 & -0.20 & 1 & -0.26 & -0.014 & -0.23 & -0.34 & -0.44 & -0.35 \\
\hline$N_{\text {eff }}$ & -0.067 & -0.32 & 0.57 & 0.71 & 0.36 & -0.21 & -0.0043 & 0.84 & 0.50 & -0.40 & 1 & 0.011 & 0.076 & 0.059 & 0.078 & 0.058 \\
\hline$f_{1}$ & -0.08 & 0.075 & 0.052 & 0.070 & -0.11 & 0.16 & 0.00070 & 0.039 & 0.028 & -0.20 & 0.064 & 1 & -0.030 & 0.051 & 0.047 & 0.031 \\
\hline$f_{2}$ & - & - & - & - & - & - & - & - & - & - & - & - & 1 & 0.10 & 0.37 & 0.23 \\
\hline$f_{3}$ & - & - & - & - & - & - & - & - & - & - & - & - & - & 1 & 0.25 & 0.36 \\
\hline$f_{4}$ & - & - & - & - & - & - & - & - & - & - & - & - & - & - & 1 & 0.11 \\
\hline$f_{5}$ & - & - & - & - & - & - & - & - & - & - & - & - & - & - & - & 1 \\
\hline
\end{tabular}




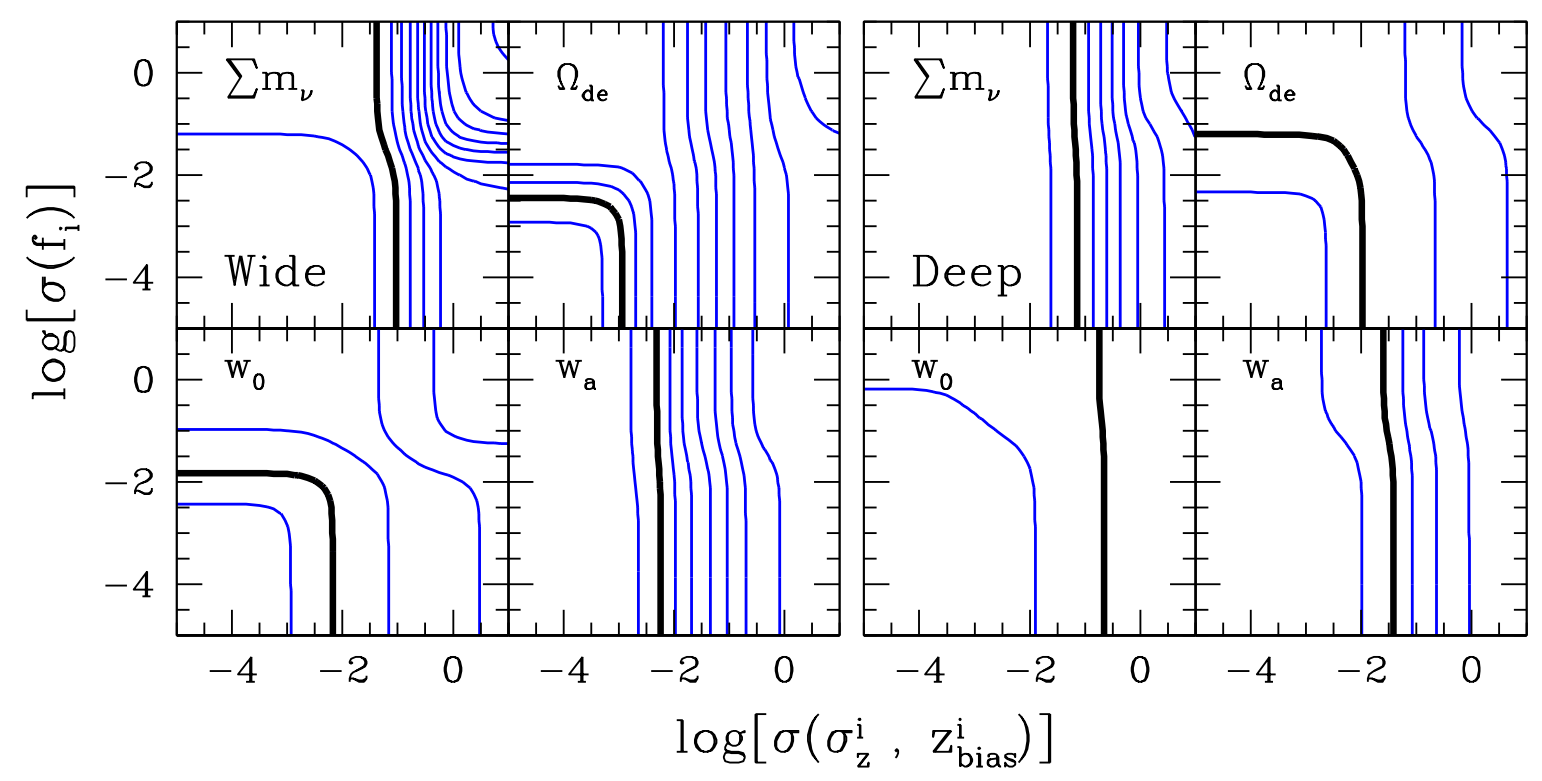

Figure 6. Degradation in the projected sensitivities, relative to the best achievable errors by a systematics-free lensing survey, as a function of priors on the shear calibration bias and photometric redshift errors. Contours of constant degradation are shown in intervals of $20 \%$, in ascending order from left/bottom to right/top. The thick black line in each plot indicates a sensitivity degradation of $30 \%$. Left: Contours for Planck+Wide-5. Right: Contours for Planck+Deep-5.

calibration bias and photometric redshift errors for two combinations of Planck and lensing tomography with five bins. Clearly, the largely vertical contours suggest that the accuracy to which the photometric redshift uncertainties can be controlled is a crucial factor in deciding a survey's parameter sensitivities. For the neutrino mass determination, for example, the sensitivity degrades rapidly as the accuracy requirement for $\left\{\sigma_{z}^{i}, z_{\text {bias }}^{i}\right\}$ is relaxed. In contrast, the degradation due to shear calibration errors saturates at $\sim 70 \%$ for $\Omega_{\mathrm{de}}$ (Planck+Wide-5), and much less for other parameters. This is qualitatively consistent with the findings of [70], where the shear calibration errors are said to enter a "self-calibration" regime.

Changing $\ell_{\max }$ to a lower value degrades further the parameter sensitivities. For the neutrino mass extraction, the degradation is $\sim 30 \%$ from $\ell_{\max }=3000$ to $\ell_{\max }=1000$. For the dark energy parameters, on the other hand, the sensitivities degrade generally by less than $20 \%$.

Focussing now on the $m_{\nu}, w$ degeneracy, Table 5 compares various projected constraints on the neutrino mass for the eleven parameter model (cf. section 4.2) and a seven parameter model which assumes $\left\{N_{\text {eff }}, \alpha_{s}, w_{0}, w_{a}\right\}$ to be fixed at $\{3,0,-1,0\}$. From Planck and weak lensing without tomography, the expected $1 \sigma$ error for the two models, $0.15 \mathrm{eV}$ and $0.082 \mathrm{eV}$, differ by almost a factor of two. With the inclusion of five tomography bins, the errors reduce to $0.043 \mathrm{eV}$ and $0.037 \mathrm{eV}$ respectively, and only a $\sim 15 \%$ discrepancy remains between them. This clearly demonstrates that weak lensing tomography can break the $m_{\nu}, w$ degeneracy very effectively. 
Table 5. Projected $1 \sigma$ constraints on the neutrino mass from various combinations of Planck and an LSST-like wide lensing survey (with one and five tomography bins) for two cosmological models. The eleven parameter model corresponds to that presented in section 4.2 , while the seven parameter model assumes $\left\{N_{\mathrm{eff}}, \alpha_{s}, w_{0}, w_{a}\right\}$ to be fixed at $\{3,0,-1,0\}$.

\begin{tabular}{llc}
\hline Model & Cosmological probes & $\sigma\left(\sum m_{\nu}\right)$ \\
\hline 11 parameters & Planck only & $0.48 \mathrm{eV}$ \\
11 parameters & Planck+Wide-1 & $0.15 \mathrm{eV}$ \\
11 parameters & Planck+Wide-5 & $0.043 \mathrm{eV}$ \\
7 parameters & Planck+Wide-1 & $0.082 \mathrm{eV}$ \\
7 parameters & Planck+Wide-5 & $0.037 \mathrm{eV}$ \\
\hline
\end{tabular}

\section{Conclusions}

Cosmological probes are already at an accuracy level to probe important aspects of particle physics such as neutrino masses and the dark energy equation of state. Current CMB, LSS, SNIa and BAO data can already pin the former down to $\sum m_{\nu} \lesssim 0.5 \mathrm{eV}$ [15]. With future CMB data and weak lensing surveys, the projected sensitivity to these parameters will be far better. From a combined analysis of prospective data from the Planck mission and a future LSST-like full-sky lensing survey, the sensitivity to $\sum m_{\nu}$ is expected to reach $0.05 \mathrm{eV}$, sufficient to probe the difference between the normal and inverted hierarchy schemes.

It is interesting to compare our results to those derived in [54], which also considers the combination of Planck data with a future weak lensing survey. For the model closest to our Planck+Wide- 5 eleven parameter scenario (cf. Table 5), they find a sensitivity of $\sigma\left(\sum m_{\nu}\right)=0.045 \mathrm{eV}$, compared to our $0.043 \mathrm{eV}$. Systematics issues in the weak lensing survey have been neglected in the analysis of [54]. The same is true in our analysis in the sense that we have assumed the systematic effects to be controlled to the accuracy needed in order not to seriously degrade the parameter determination.

A similar sensitivity to $\sum m_{\nu}$ can also be reached by combining Planck data with future cluster abundance measurements [99], $\sigma\left(\sum m_{\nu}\right)=0.034 \mathrm{eV}$, and high-redshift galaxy surveys [100], $\sigma\left(\sum m_{\nu}\right)=0.059 \mathrm{eV}(0.5<z<2)$ and $0.043 \mathrm{eV}(2<z<4)$. Note that these numbers were derived in $[99,100]$ for an equivalent of our seven parameter model. However, since both methods provide a measure of the distance-redshift relation, and therefore share the same principle with weak lensing tomography, it is very probable that these alternative probes could also offer some improvement towards the degeneracy between the neutrino mass and the dark energy equation of state, should the latter have been treated as a free parameter. This remains to be verified.

A partial breakage of the said degeneracy is also possible by cross-correlating CMB and LSS measurements. The analysis of [101] finds that the combination of Planck and future LSS data, including cross-correlation between the two data sets, can bring the $\sum m_{\nu}$ sensitivity to the $0.1 \mathrm{eV}$ level. This, however, is not competitive with what is 
expected from weak lensing surveys.

Finally, the weak lensing signal can be extracted from the CMB data itself. With data from the Planck satellite, the authors of [102] estimate that the neutrino mass can be determined to $\sigma\left(\sum m_{\nu}\right)=0.51 \mathrm{eV}$ without lensing extraction, a number comparable to our $0.48 \mathrm{eV}$ (cf. Table 5). With lensing extraction the error reduces to $0.15 \mathrm{eV}$, i.e., a factor of three improvement. Clearly, CMB lensing adds some of the same information gained in a galaxy lensing survey, except that the source redshift is locked at $z \sim 1100$. Therefore, a CMB survey with a much higher resolution is needed in order to breach the $\sigma\left(\sum m_{\nu}\right)=0.1 \mathrm{eV}$ barrier for the neutrino mass determination. With the proposed Inflation Probe mission, the analysis of [102] finds that the sensitivity could reach $0.04 \mathrm{eV}$ if lensing extraction is included, a precision similar to that attainable by large scale tomographic galaxy lensing surveys found in our analysis. The combination of these techniques could plausibly lead to even better sensitivities.

\section{Acknowledgements}

We thank Håkon Dahle and Bjarne Thomsen for enlightening discussions on the systematics related to weak lensing surveys.

\section{Appendix A. The halo model}

The halo model supposes that all matter in the universe is partitioned into distinct units, the halos. This assumption allows one to study the universal matter distribution in two steps: the distribution of matter within each halo, and the spatial distribution of the halos themselves. In its simplest application, the halo model proposes that the matter power spectrum $P^{\mathrm{NL}}(k, z)$ be composed of two distinct terms (e.g., [103]),

$$
P^{\mathrm{NL}}(k, z)=P^{1-\text { halo }}(k, z)+P^{2-\text { halo }}(k, z),
$$

where

$$
\begin{aligned}
& P^{1-\text { halo }}(k, z)=\int d M n(M, z)\left(\frac{M}{\bar{\rho}_{\text {halo }}}\right)^{2}|u(k \mid M)|^{2}, \\
& P^{2-\text { halo }}(k, z)=P^{\mathrm{L}}(k, z)\left[\int d M n(M, z) b(M)\left(\frac{M}{\bar{\rho}_{\text {halo }}}\right) u(k \mid M)\right]^{2},
\end{aligned}
$$

describe, respectively, the correlations between two points drawn from the same halo and from two different halos. The former dominates on small scales, while the latter rises to prominence on large scales and approaches the power spectrum calculated from linear theory $P^{\mathrm{L}}(k, z)$ as $k \rightarrow 0$. The average matter density $\bar{\rho}_{\text {halo }}$ counts all matter clustered in halos. For a basic $\Lambda$ CDM set-up, $\bar{\rho}_{\text {halo }}$ is well approximated by $\bar{\rho}_{\text {halo }} \simeq \bar{\rho}_{m} \equiv \Omega_{m} \rho_{\text {crit }}$, where $\rho_{\text {crit }}$ is the present critical density.

Three pieces of information are required to complete the model.

(i) The mass function $n(M, z)$, usually written as $n(M, z) d M=\bar{\rho}_{\text {halo }} / M f(\nu) d \nu$, specifies the number density of halos of mass $M$ at redshift $z$. Here, $f(\nu)$ is a 
universal function of the peak height $\nu=\delta_{\mathrm{sc}}^{2} / \sigma_{\mathrm{L}}^{2}(M, z)$, where $\delta_{\mathrm{sc}}=1.68$ is the linear overdensity at the epoch of spherical collapse, and

$$
\sigma_{\mathrm{L}}^{2}(M, z) \equiv \int \frac{d^{3} k}{(2 \pi)^{3}} P^{\mathrm{L}}(k, z)|W(k R)|^{2}
$$

is the rms linear fluctuations filtered with a tophat function $W(x)=3 / x^{3}(\sin x-$ $x \cos x)$ on a scale of $R=\left(3 M / 4 \pi \bar{\rho}_{m}\right)^{1 / 3}$. A number of variants for $f(\nu)$ exists in the literature. Here, we adopt the version proposed by Sheth and Tormen [104].

(ii) The linear bias $b(\nu)$ parameterises the clustering strength of halos relative to the underlying dark matter, and obeys $\int d \nu f(\nu) b(\nu)=1$ by construction.

(iii) The function $u(k \mid M)$ is the Fourier transform of the matter distribution $\rho(r \mid M)$ within a halo, normalised to the halo mass $M$. A natural choice for $\rho(r \mid M)$ would be the Navarro-Frenk-White profile [105, 106].

To generalise the halo model to mixed dark matter cosmologies, we assume that only baryons and CDM cluster in halos, while the neutrinos remain an almost smooth component. Thus, equations (A.1) to (A.3) are evaluated for $P^{\mathrm{L}}(k, z)=P_{b+c}^{\mathrm{L}}(k, z)$, where $P_{b+c}^{\mathrm{L}}(k, z)$ is the combined baryon and CDM linear power spectrum defined in (3.5). Correspondingly, we take the average matter density in halos to be $\bar{\rho}_{\text {halo }} \simeq$ $\left(\Omega_{b}+\Omega_{c}\right) \rho_{\text {crit }}$. The corrected spectrum $P_{b+c}^{\mathrm{NL}}(k, z)$ is then added with the linear neutrino spectrum $P_{\nu}^{\mathrm{L}}(k, z)$ to form the total matter power spectrum $P_{m}(k, z)$ as per $(3.4)$.

\section{References}

[1] A. G. Riess et al. [Supernova Search Team Collaboration], Astron. J. 116 (1998) 1009 [arXiv:astro$\mathrm{ph} / 9805201]$.

[2] S. Perlmutter et al. [Supernova Cosmology Project Collaboration], Astrophys. J. 517 (1999) 565 [arXiv:astro-ph/9812133].

[3] D. N. Spergel et al. [WMAP Collaboration], Astrophys. J. Suppl. 148 (2003) 175 [arXiv:astro$\mathrm{ph} / 0302209]$.

[4] M. Tegmark et al. [SDSS Collaboration], Phys. Rev. D 69 (2004) 103501 [arXiv:astro-ph/0310723].

[5] G. L. Fogli, E. Lisi, A. Marrone and A. Palazzo, arXiv:hep-ph/0506083.

[6] S. Hannestad, arXiv:hep-ph/0602058.

[7] S. Hannestad, JCAP 0305 (2003) 004 [arXiv:astro-ph/0303076].

[8] Ø. Elgarøy and O. Lahav, New J. Phys. 7 (2005) 61 [arXiv:hep-ph/0412075].

[9] V. Barger, D. Marfatia and A. Tregre, Phys. Lett. B 595 (2004) 55 [arXiv:hep-ph/0312065].

[10] P. Crotty, J. Lesgourgues and S. Pastor, Phys. Rev. D 69 (2004) 123007 [arXiv:hep-ph/0402049].

[11] U. Seljak et al., Phys. Rev. D 71 (2005) 103515 [arXiv:astro-ph/0407372].

[12] G. L. Fogli, E. Lisi, A. Marrone, A. Melchiorri, A. Palazzo, P. Serra and J. Silk, Phys. Rev. D 70 (2004) 113003 [arXiv:hep-ph/0408045].

[13] M. Tegmark, Phys. Scripta T121 (2005) 153 [arXiv:hep-ph/0503257].

[14] S. Hannestad and G. Raffelt, JCAP 0404 (2004) 008 [arXiv:hep-ph/0312154].

[15] A. Goobar, S. Hannestad, E. Mörtsell and H. Tu, JCAP 0606 (2006) 019 [arXiv:astro-ph/0602155].

[16] C. Weinheimer, Nucl. Phys. Proc. Suppl. 118 (2003) 279.

[17] V. M. Lobashev et al., Nucl. Phys. Proc. Suppl. 91 (2001) 280.

[18] A. Osipowicz et al. [KATRIN Collaboration], arXiv:hep-ex/0109033.

[19] http://www-ik.fzk.de/ katrin/index.html

[20] R. Bean and A. Melchiorri, Phys. Rev. D 65 (2002) 041302 [arXiv:astro-ph/0110472]. 
[21] S. Hannestad and E. Mörtsell, Phys. Rev. D 66 (2002) 063508 [arXiv:astro-ph/0205096].

[22] A. Melchiorri, L. Mersini-Houghton, C. J. Odman and M. Trodden, Phys. Rev. D 68 (2003) 043509 [arXiv:astro-ph/0211522].

[23] P. Astier et al., Astron. Astrophys. 447 (2006) 31 [arXiv:astro-ph/0510447].

[24] S. Hannestad and E. Mörtsell, JCAP 0409 (2004) 001 [arXiv:astro-ph/0407259].

[25] Y. Wang and M. Tegmark, Phys. Rev. Lett. 92 (2004) 241302 [arXiv:astro-ph/0403292].

[26] A. Upadhye, M. Ishak and P. J. Steinhardt, Phys. Rev. D 72 (2005) 063501 [arXiv:astro$\mathrm{ph} / 0411803]$.

[27] C. Wetterich, Nucl. Phys. B 302 (1988) 668.

[28] B. Ratra and P. J. E. Peebles, Phys. Rev. D 37 (1988) 3406.

[29] P. J. E. Peebles and B. Ratra, Astrophys. J. 325 (1988) L17.

[30] C. Deffayet, G. R. Dvali and G. Gabadadze, Phys. Rev. D 65 (2002) 044023 [arXiv:astro$\mathrm{ph} / 0105068]$.

[31] G. Dvali and M. S. Turner, arXiv:astro-ph/0301510.

[32] S. Hannestad, Phys. Rev. Lett. 95 (2005) 221301 [arXiv:astro-ph/0505551].

[33] D. J. Eisenstein et al., Astrophys. J. 633 (2005) 560 [arXiv:astro-ph/0501171].

[34] M. Bartelmann and P. Schneider, Phys. Rept. 340 (2001) 291 [arXiv:astro-ph/9912508].

[35] L. Van Waerbeke and Y. Mellier, arXiv:astro-ph/0305089.

[36] A. Refregier, Ann. Rev. Astron. Astrophys. 41 (2003) 645 [arXiv:astro-ph/0307212].

[37] L. van Waerbeke et al., Astron. Astrophys. 358 (2000) 30 [arXiv:astro-ph/0002500].

[38] N. Kaiser, G. Wilson and G. A. Luppino, arXiv:astro-ph/0003338.

[39] D. J. Bacon, A. R. Refregier and R. S. Ellis, Mon. Not. Roy. Astron. Soc. 318 (2000) 625 [arXiv:astro-ph/0003008].

[40] D. M. Wittman, J. A. Tyson, D. Kirkman, I. Dell'Antonio and G. Bernstein, Nature 405 (2000) 143 [arXiv:astro-ph/0003014].

[41] H. Hoekstra et al., arXiv:astro-ph/0511089.

[42] E. Semboloni et al., Astron. Astrophys. 452 (2006) 51 [arXiv:astro-ph/0511090].

[43] http://www.cfht.hawaii.eud/Science/CFHTLS/

[44] T. Abbott et al. [Dark Energy Survey Collaboration], arXiv:astro-ph/0510346.

[45] http://pan-starrs.ifa.hawaii.edu/

[46] http://www.vista.ac.uk/

[47] http://snap.lbl.gov/

[48] http://www.lsst.org/

[49] W. Hu, Astrophys. J. 522 (1999) L21 [arXiv:astro-ph/9904153].

[50] D. Huterer, Phys. Rev. D 65 (2002) 063001 [arXiv:astro-ph/0106399].

[51] W. Hu, Phys. Rev. D 66 (2002) 083515 [arXiv:astro-ph/0208093].

[52] K. N. Abazajian and S. Dodelson, Phys. Rev. Lett. 91 (2003) 041301 [arXiv:astro-ph/0212216].

[53] M. Ishak, C. M. Hirata, P. McDonald and U. Seljak, Phys. Rev. D 69 (2004) 083514 [arXiv:astro$\mathrm{ph} / 0308446]$.

[54] Y. S. Song and L. Knox, Phys. Rev. D 70 (2004) 063510 [arXiv:astro-ph/0312175].

[55] M. Takada and B. Jain, Mon. Not. Roy. Astron. Soc. 348 (2004) 897 [arXiv:astro-ph/0310125].

[56] M. Ishak, Mon. Not. Roy. Astron. Soc. 363 (2005) 469 [arXiv:astro-ph/0501594].

[57] R. Massey et al., Astron. J. 127 (2004) 3089 [arXiv:astro-ph/0304418].

[58] A. Refregier et al., Astron. J. 127 (2004) 3102 [arXiv:astro-ph/0304419].

[59] N. Kaiser, Astrophys. J. 388 (1992) 272.

[60] N. Kaiser, Astrophys. J. 498 (1998) 26 [arXiv:astro-ph/9610120].

[61] S. Dodelson, Modern cosmology, Academic Press, Amsterdam, 2003.

[62] L. Van Waerbeke, Y. Mellier and H. Hoekstra, Astron. Astrophys. 429 (2005) 75 [arXiv:astro$\mathrm{ph} / 0406468]$.

[63] M. Jarvis and B. Jain, arXiv:astro-ph/0412234.

[64] L. M. Wang and P. J. Steinhardt, Astrophys. J. 508 (1998) 483 [arXiv:astro-ph/9804015]. 
[65] A. Fernandez-Soto, K. M. Lanzetta and A. Yahil, Astrophys. J. 513 (1999) 34 [arXiv:astro$\mathrm{ph} / 9809126]$.

[66] M. L. Brown, A. N. Taylor, D. J. Bacon, M. E. Gray, S. Dye, K. Meisenheimer and C. Wolf, Mon. Not. Roy. Astron. Soc. 341 (2003) 100 [arXiv:astro-ph/0210213].

[67] M. Davis et al., Proc. SPIE 4834 (2003) 161 [arXiv:astro-ph/0209419].

[68] O. Le Fevre et al. [The VIMOS VLT Deep Survey Collaboration], Astron. Astrophys. 428 (2004) 1043 [arXiv:astro-ph/0403628].

[69] Z. M. Ma, W. Hu and D. Huterer, Astrophys. J. 636 (2005) 21 [arXiv:astro-ph/0506614].

[70] D. Huterer, M. Takada, G. Bernstein and B. Jain, Mon. Not. Roy. Astron. Soc. 366 (2006) 101 [arXiv:astro-ph/0506030].

[71] M. Ishak and C. M. Hirata, Phys. Rev. D 71 (2005) 023002 [arXiv:astro-ph/0405042].

[72] C. Heymans et al., Mon. Not. R. Astron. Soc. 368 (2006) 1323. [arXiv:astro-ph/0506112].

[73] C. M. Hirata and U. Seljak, Mon. Not. Roy. Astron. Soc. 343 (2003) 459 [arXiv:astro-ph/0301054].

[74] H. Dahle, private communication (2006).

[75] B. Jain and U. Seljak, Astrophys. J. 484 (1997) 560 [arXiv:astro-ph/9611077].

[76] U. Seljak and M. Zaldarriaga, Astrophys. J. 469 (1996) 437; http://www . cmbfast. org/.

[77] K. Heitmann, P. M. Ricker, M. S. Warren and S. Habib, Astrophys. J. Suppl. 160 (2005) 28 [arXiv:astro-ph/0411795].

[78] D. Huterer and M. Takada, Astropart. Phys. 23 (2005) 369 [arXiv:astro-ph/0412142].

[79] A. Klypin, A. V. Maccio, R. Mainini and S. A. Bonometto, Astrophys. J. 599 (2003) 31 [arXiv:astro-ph/0303304].

[80] E. V. Linder and A. Jenkins, Mon. Not. Roy. Astron. Soc. 346 (2003) 573 [arXiv:astro-ph/0305286].

[81] K. Dolag, M. Bartelmann, F. Perrotta, C. Baccigalupi, L. Moscardini, M. Meneghetti and G. Tormen, Astron. Astrophys. 416 (2004) 853 [arXiv:astro-ph/0309771].

[82] M. Bartelmann, K. Dolag, F. Perrotta, C. Baccigalupi, L. Moscardini, M. Meneghetti and G. Tormen, New Astron. Rev. 49 (2005) 199 [arXiv:astro-ph/0404489].

[83] J. A. Peacock and S. J. Dodds, Mon. Not. Roy. Astron. Soc. 280 (1996) L19 [arXiv:astro$\mathrm{ph} / 9603031]$.

[84] U. Seljak, Mon. Not. Roy. Astron. Soc. 318 (2000) 203 [arXiv:astro-ph/0001493].

[85] J. A. Peacock and R. E. Smith, Mon. Not. Roy. Astron. Soc. 318 (2000) 1144 [arXiv:astro$\mathrm{ph} / 0005010]$.

[86] C. P. Ma and J. N. Fry, Astrophys. J. 543 (2000) 503, [arXiv:astro-ph/0003343].

[87] R. E. Smith et al. [The Virgo Consortium Collaboration], Mon. Not. Roy. Astron. Soc. 341 (2003) 1311 [arXiv:astro-ph/0207664].

[88] M. J. White and C. Vale, Astropart. Phys. 22 (2004) 19 [arXiv:astro-ph/0312133].

[89] A. Ringwald and Y. Y. Y. Wong, JCAP 0412 (2004) 005 [arXiv:hep-ph/0408241].

[90] K. Abazajian, E. R. Switzer, S. Dodelson, K. Heitmann and S. Habib, Phys. Rev. D 71 (2005) 043507 [arXiv:astro-ph/0411552].

[91] S. Hannestad, A. Ringwald, H. Tu and Y. Y. Y. Wong, JCAP 0509 (2005) 014 [arXiv:astro$\mathrm{ph} / 0507544]$.

[92] M. White, Astropart. Phys. 22 (2004) 211 [arXiv:astro-ph/0405593].

[93] H. Zhan and L. Knox, Astrophys. J. 616 (2004) L75 [arXiv:astro-ph/0409198].

[94] Y. P. Jing, P. Zhang, W. P. Lin, L. Gao and V. Springel, Astrophys. J. 640 (2006) L119 [arXiv:astro-ph/0512426].

[95] http://astro.estec.esa.nl/Planck/

[96] M. Chevallier and D. Polarski, Int. J. Mod. Phys. D 10 (2001) 213 [arXiv:gr-qc/0009008].

[97] E. V. Linder, Phys. Rev. Lett. 90 (2003) 091301 [arXiv:astro-ph/0208512].

[98] J. Lesgourgues, S. Pastor and L. Perotto, Phys. Rev. D 70 (2004) 045016 [arXiv:hep-ph/0403296].

[99] S. Wang, Z. Haiman, W. Hu, J. Khoury and M. May, Phys. Rev. Lett. 95 (2005) 011302 [arXiv:astro-ph/0505390].

[100] M. Takada, E. Komatsu and T. Futamase, Phys. Rev. D 73 (2006) 083520 [arXiv:astro- 

ph/0512374].

[101] K. Ichikawa and T. Takahashi, arXiv:astro-ph/0510849.

[102] J. Lesgourgues, L. Perotto, S. Pastor and M. Piat, Phys. Rev. D 73 (2006) 045021 [arXiv:astroph/0511735].

[103] A. Cooray and R. Sheth, Phys. Rept. 372 (2002) 1 [arXiv:astro-ph/0206508].

[104] R. K. Sheth and G. Tormen, Mon. Not. Roy. Astron. Soc. 308 (1999) 119 [arXiv:astroph/9901122].

[105] J. F. Navarro, C. S. Frenk and S. D. M. White, Astrophys. J. 462 (1996) 563 [arXiv:astroph/9508025];

[106] J. F. Navarro, C. S. Frenk and S. D. M. White, Astrophys. J. 490 (1997) 493. 